\title{
Biocultural key species: an assembly of biotic resources with biocultural importance
}

\section{Tania Vianney Gutiérrez-Santillán ( $\nabla$ tvgutierrez_santillan@yahoo.com.mx )}

Universidad Autonónoma de Nuevo León, Facultad de Ciencias Forestales https://orcid.org/00000002-7784-3167

\section{Eduardo Estrada-Castillón}

Universidad Autónoma de Nuevo León: Facultad de Ciencias Forestales

Gerardo Sánchez-Rojas

Universidad Autónoma del Estado de Hidalgo: Centro de Investigaciones Biológicas

Jorge Valencia-Herverth

Tecnológico Nacional de México: Instituto Tecnológico de Huejutla: Extensión Molango

Luis Gerardo Cuellar-Rodríguez

Universidad Autónoma de Nuevo León: Universidad Autonoma de Nuevo Leon

Jaime Sánchez-Salas

Universidad Juárez del Estado de Durango: Facultad de Ciencias Biológicas

\section{Daniel Robles-García}

Universidad Autónoma del Estado de Hidalgo: Centro de Investigaciones Biológicas

\section{Víctor Abraham Vargas-Vázquez}

Ecological Research Azcatl

\section{Francisco Reyes-Zepeda}

Universidad Autónoma de Tamaulipas: Instituto de Ecología Aplicada

\section{Rodolfo Torres de los Santos}

Universidad Autónoma de Tamaulipas: Unidad Académica Multidisciplinaria Mante

\section{Eduardo Lara-Reimers}

Universidad Autónoma Agraria Antonio Narro: Departamento Forestal

\section{Diana Uresti-Durán}

Instituto Nacional de Investigaciones Forestales, Agrícolas y Pecuarias: Campo Experimental Saltillo

\section{Gisela Muro-Pérez}

Universidad Juárez del Estado de Durango: Facultad de Ciencias Biológicas

\section{Gamaliel Castañeda-Gaytán}

Universidad Juárez del Estado de Durango: Facultad de Ciencias Biológicas

\section{David Figueroa-Serrano}

Universidad Autónoma del Estado de México: Facultad de Antropología

Daniel Hernández-Ramírez

Instituto Tecnológico de Zacatecas: Instituto Tecnologico de Zacatecas 


\section{Research Article}

Keywords: Cultural importance indexes, traditional ecological knowledge, Náhuatl, Huastec, Mexico

Posted Date: August 24th, 2021

DOI: https://doi.org/10.21203/rs.3.rs-399431/v1

License: (c) (i) This work is licensed under a Creative Commons Attribution 4.0 International License. Read Full License 


\section{Biocultural key species: an assembly of biotic resources with biocultural importance}

${ }^{1 *}$ Tania Vianney Gutiérrez-Santillán, ${ }^{1 *}$ Andrés Eduardo Estrada-Castillón, ${ }^{2}$ Gerardo Sánchez-Rojas, ${ }^{3}$ Jorge Valencia-Herverth, ${ }^{1}$ Luis Gerardo Cuéllar-Rodríguez ${ }^{4} \mathrm{Jaime}$ Sánchez-Salas, ${ }^{2}$ Daniel Robles-García, ${ }^{5}$ Víctor Abraham Vargas-Vázquez, ${ }^{6}$ Francisco Reyes-Zepeda, ${ }^{7}$ Rodolfo Torres de los Santos, ${ }^{8}$ Eduardo Lara-Reimers, ${ }^{9}$ Diana UrestiDurán, ${ }^{4}$ Gisela Muro-Pérez, ${ }^{4}$ Gamaliel Castañeda-Gaytán, ${ }^{10}$ David Figueroa-Serrano, ${ }^{11}$ Daniel Hernández-Ramírez

${ }^{1}$ Universidad Autónoma de Nuevo León, Facultad de Ciencias Forestales. Carr. Nacional Linares-Cd. Victoria km 145, A.P. 41, 6700. Linares, Nuevo León, México.

${ }^{2}$ Universidad Autónoma del Estado de Hidalgo, Centro de Investigaciones Biológicas. Carr. Pachuca-Tulancingo km 4.5, Col. Carboneras, C.P. 42184. Pachuca de Soto, Hidalgo, México.

${ }^{3}$ Tecnológico Nacional de México, Instituto Tecnológico de Huejutla, Extensión Molango, Av. Corregidora sin número, Barrio Santa Cruz Primera Sección, C.P. 43100. Molango de Escamilla, Hidalgo, México.

${ }^{4}$ Universidad Juárez del Estado de Durango, Facultad de Ciencias Biológicas. Av. Universidad sin número, Fracc. Filadelfia, C.P. 35010. Gómez Palacio, Durango, México.

${ }^{5}$ Ecological Research Azcatl SAS, Allende 901 Héroe de Nacozari, CP. 87030. Ciudad Victoria, Tamaulipas, México.

${ }^{6}$ Universidad Autónoma de Tamaulipas, Instituto de Ecología Aplicada. Av. División del Golfo 356 Col. Libertad, C.P. 87020. Ciudad Victoria, Tamaulipas, México.

${ }^{7}$ Universidad Autónoma de Tamaulipas, Unidad Académica Multidisciplinaria Mante Centro. Blvd. Enrique Cárdenas González 1201 Pte. Col. Jardín. C.P. 89840. Ciudad Mante, Tamaulipas, México.

${ }^{8}$ Universidad Autónoma Agraria Antonio Narro, Departamento Forestal. Calz. Antonio Narro 1923, Buenavista, C.P. 25315; Saltillo, Coahuila, México.

${ }^{9}$ Instituto Nacional de Investigaciones Forestales, Agrícolas y Pecuarias, Campo Experimental Saltillo. Carr. Saltillo-Zacatecas km 342+119, 9515, Hacienda de Buenavista, C.P. 25315. Saltillo, Coahuila, México. 
${ }^{10}$ Universidad Autónoma del Estado de México, Facultad de Antropología. Mariano Matamoros 1065A, C.P. 50130. Toluca, Estado de México, México.

${ }^{11}$ Instituto Regional Patrimonio Mundial en Zacatecas, Centro Categoría Bajos los Auspicios de la UNESCO. Av. Juan de Tolosa 831, Centro Histórico, C.P. 98000. Zacatecas, México.

Corresponding Author: tvgutierrez_santillan@yahoo.com.mx;

aeduardoestradac@prodigy.net.mx

\section{Declarations}

Ethics approval and consent to participate: "In each interview, the informant's consent was obtained, including the consent to take photographs and participant observation activities (International Society of Ethnobiology 2006; http://ethnobiology.net/codeofethics/)"

Consent for publication: Not applicable

Availability of data and materials: Not applicable, "Data sharing not applicable to this article as no datasets were generated or analused durin the current study"

Competing interests: "The authors declare that they have no competing interests"

Funding: Not applicable

Authors' Contributions: Not applicable 
Acknowledgements: To the people of the community who contributed their local knowledge about the biocultural diversity of the region.

Authors' information: Not aplicable

\begin{abstract}
Background: The cultural importance assigned to biodiversity is a complex subject, its study has been developed from ethnobotany and ethnomicology, mainly. A new proposal to address cultural importance is from the biocultural key species. Therefore, integrating information on the knowledge and use of various biological groups, the species with the greatest cultural relevance were selected.

Methods: An index (BKSI: Biocultural Key Species Index) was integrated, which assesses the relevance based on general attributes associated with fauna, flora and funga. The study was carried out in the Náhuatl community called El Barco, Lolotla; in the Huastec Hidalguense region. Field work was carried out for two years (2018-2019) with 24 field trips. Percentage ethnographic method combined with snowball (10\% of the population) were applied; and as the ethnographic tool multiple free listings $(\mathrm{n}=50)$ were used.

Results: In total of 335 species associated to 537 traditional names in Spanish and indigenous language (Náhuatl) are reported, that belongs to different biological groups (fungi and plants; fish, amphibians, reptiles, birds and mammals). The biocultural relevance degree was categorized respect to the obtained value in the index (BKSI); a representative set of each biological group was selected, being those with the highest biocultural relevance.

Conclusions: It is proposed that these species constitute a useful tool in the application of programs that promote the conservation of diversity from a biocultural approach; they may even promote the proper use of resources or biological restoration.
\end{abstract}

\title{
Key word
}

Cultural importance indexes; traditional ecological knowledge; Náhuatl, Huastec, Mexico.

\section{Resumen}


Antecedentes: La importancia cultural asignada a la biodiversidad es un tema complejo, su estudio se ha desarrollado desde la etnobotánica y la etnomicología, principalmente. Una nueva propuesta para abordar la importancia cultural es la de las especies clave bioculturales. Por lo tanto, integrando información sobre el conocimiento y uso de diversos grupos biológicos, se seleccionaron las especies con mayor relevancia cultural.

Método: Se integró un índice (IEBC: Índice de Especies Bioculturales Clave), que evalúa la relevancia con base en atributos generales asociados a la fauna, flora y hongos. El estudio se realizó en la comunidad Náhuatl denominada El Barco, Lolotla; en la región Huasteca Hidalguense. Se realizó trabajo de campo durante dos años (2018-2019) con 24 salidas de campo. Se aplicó el método etnográfico porcentual combinado con bola de nieve ( $10 \%$ de la población); y como herramienta etnográfica se utilizaron listados libres múltiples $(\mathrm{n}=50)$. Resultados: En total se reportan 335 especies asociadas a 537 nombres tradicionales en español y lengua indígena (Náhuatl), que pertenecen a diferentes grupos biológicos (hongos

y plantas; peces, anfibios, reptiles, aves y mamíferos). El grado de relevancia biocultural se categorizó con respecto al valor obtenido en el índice. Se seleccionó un conjunto representativo de cada grupo biológico, siendo los de mayor relevancia biocultural.

Conclusión: Se propone que estas especies constituyan una herramienta útil en la aplicación de programas que promuevan la conservación de la diversidad desde un enfoque biocultural; incluso pueden promover el uso adecuado de los recursos o la restauración biológica.

\section{Palabras clave}

Índices de importancia cultural; conocimiento ecológico tradicional; Náhuatl, Huasteca, México.

\section{Background}

Cultural importance has been defined as the value that a biotic resource plays within a culture [1]. This concept is strongly related to the value that a certain culture or society assigns to it, that is, the way in which it is perceived, known and used. Cultural importance is relative and can be quite complex, especially for those resources that have a participation in different roles or cultural domains. There are different ways to approach to the cultural importance, for example, through the documentation of traditional nomenclature [2-4]; traditional 
knowledge [5,6]; nature-culture relationship [7-8]; as well as the methodological approaches that quantify knowledge and use [9-13].

Making a significant approach to the cultural importance that human groups give to natural resources has been a priority of ethnobiology, mainly from ethnobotany and ethnomycology. Designing indexes that allow quantifying qualitative data and provides a metric that reflects cultural importance [9-16].

The conceptualization of cultural key species gives a new perspective to the approach of cultural importance [17-20], as an adaptation of Paine's ecological concept of key species [21], who defines it as "that which maintains a structure and function in an ecosystem". From this concept Garibaldi and Turner [17] developed an analogous concept to define the cultural key species, establishing them as "those that function as a cultural icon, that form the contextual culture bases, as a direct reflection of the role they play, that they possess a preponderant place in the language, ceremonies and narrations". The designation of a cultural key species underlies its cultural importance, being its main characteristic its embedding in the culture linking socio-ecological systems.

Despite the controversy of the analogous adjustment of the concept of ecological key species to ethnobiology [22], the idea of cultural key species continues to be supported. Platten and Henfrey [19] define them "as a complex made up of more than one biological species, sometimes it may only be one representative, but generally it is not an isolated species, they are strongly associated with a complex of knowledge, techniques, beliefs, actions, perceptions, uses; they are generally closely related to subsistence activities". The authors consider that cultural importance, strictly speaking in how ethnobiology conceptualizes and quantifies these species, does not determine them; because it is necessary to describe the flows that it possesses at a certain level of biocultural organization.

Ellen [18] calls them ethnobiologically key species and Ibarra et al. [20] as biocultural key species. The importance of identifying these species is that they can be considered as an interdisciplinary tool as a guide in the practice of conservation biology [19]. It is known that conservation programs establishment that involve species with some degree of cultural importance are more successful than those promoted solely by government policies [23]. And even more if we speak in terms of promoting the conservation of biocultural diversity, as a 
relatively new trend that proposes the comprehensive recognition and conservation of socioecological relationships and biodiversity [25-26].

Combining the different terms to define and name this type of species, it is proposed to use the term biocultural key species [20]. Based on the aforementioned conceptual proposals, we define them as: "those species that function as icons and can constitute cultural bases at different scales (local, regional or national), have a prominent place in cultural roles or domains (anthropocentric categories), can be represented by a species, but generally it is a set or assembly; because you can have representative species of different taxonomic ranges; and they are strongly related at the cognitive and pragmatic level on certain spatio-temporal scales. They must maintain the species/ethnospecies relationship as a real ethnobiological entity, understood as the correlation between taxonomic identification (species) and its cultural correspondent, which is generally equivalent to its traditional name or cultural assignment".

Cultural importance is a very complex subject from which we are far from understanding how it works, due to its complexity and nature that varies depending on the cosmovision, the local biota, beliefs, education, technological access, level of development of the communities, infrastructure, access to public health, economic activity, commerce, religion, uses and customs, among multiple variables; of people from the same place and the dynamics with their environment. However, the methodological approach that ethnobiology proposes for its understanding is a tool that brings us closer to its approach. For this reason, in this work the term of cultural importance is replaced by biocultural relevance, being understood as the value that a human group assigns to a species/ethnospecies or a set of these.

The methodological approach of cultural importance from ethnobiology helps to select species at a quantitative level important for a certain place and human group. Therefore, based on the traditional methodological conjugation and the new concepts, an index can be designed that provides a metric for its identification and selection. Based on a case study carried out in the Huastec area, in the state of Hidalgo, Mexico (with the native group Náhuatl), the known, named and used biodiversity was documented, a general attribute index was designed for all biological groups (fungi and plants; fish, amphibians, reptiles, birds and mammals) integrated by some already proposed sub-indexes and complemented with some that were designed; the index was applied and the set of biodiversity of greatest biocultural 
relevance was quantitatively determined. The index includes five variables, which are: frequency of mention by rank-abundance $\left(F_{R-A}\right)$, average mention order (AMO), richness of traditional names (RTN), value of total uses/parts used (VTU/PU) and origin of the species (O). The present work contributes to the topic of cultural relevance and biocultural key species, making an integration between both concepts and proposing a standard and simple metric that provides a quick (quantitative) approach to the selection of species/ethnospecies more relevant at biocultural level.

\section{Methods}

\subsection{Study area}

The Huastec belongs to the historical Mesoamerican region [27]; culturally it is characterized by the confluence of various indigenous groups [28]; being Náhuatl the majority group [29]. The Huastec has a rugged topography derived from its geological formation, with elevations ranging from sea level to over 2,000 meters above sea level; which directly influences a variation of climates and high biological diversity [30]. The community of El Barco, Lolotla, Hidalgo (-98.7213888889021.1772222222, 166 meters above sea level; Datum WGS84) is located in the coastal plain of the gulf at an altitude of 140 meters above sea level, with an average annual temperature of $18^{\circ} \mathrm{C}-25^{\circ} \mathrm{C}$ and an average annual rainfall of $1600-1900 \mathrm{~mm}$, the type of vegetation corresponds to sub-evergreen medium forest (S-EMF; [31]), the community is settled on the banks of the Claro River. The number of inhabitants is 372 , distributed in 75 houses, where $75 \%$ are bilingual and $25 \%$ only speak their mother language ([32]; Figure 1).

\subsection{Field work and species taxonomic identification}

The community of El Barco was visited for two years (January 2018 to December 2019) with a total of 24 field trips. The criteria of selection were by their type of vegetation, geographic location, degree of cover vegetation, roads to access the community and a high percentage of speakers of the indigenous language (Náhuatl, [32]). The project was previously presented to local authorities and approved through a community assembly for its development. Also,

free informed consent was obtained prior at each interview (International Society of 
Ethnobiology 2006; http://ethnobiology.net/code-of-ethics/; [33]), as well as taking photographs and activities with participant observation [34].
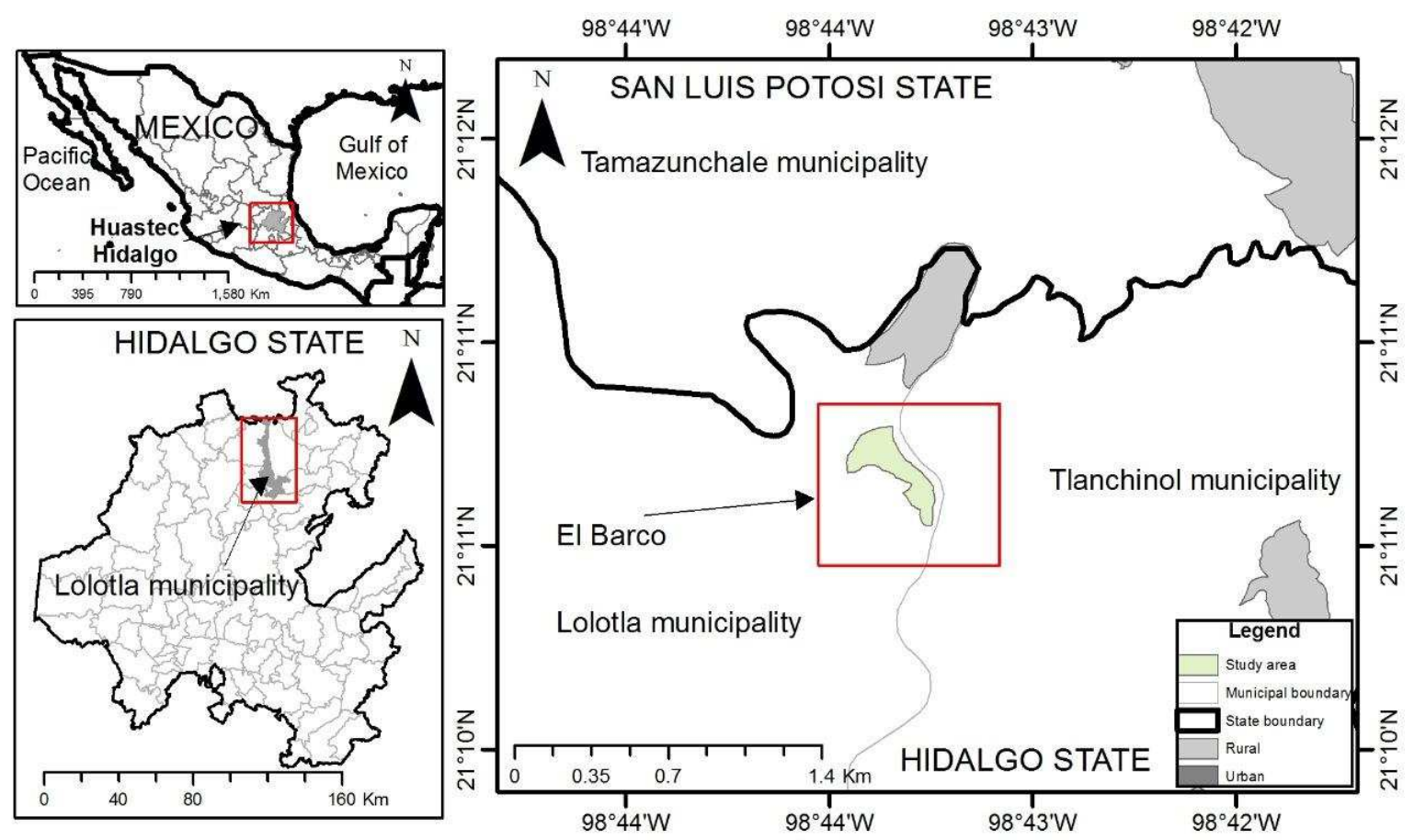

Figure 1. Spatial location of the community of El Barco, Lolotla, Hidalgo, Mexico. The community is settled in the cultural region called Huastec, the majority indigenous group is Nahuas. The region is predominant for its warm-humid climate.

The ethnographic method (percentage) was interviewing more than $10 \%(n=50)$ of the local population [35], and the selection of the informants was by the snowball technique [36], with an age range of 17 to 80 years old. Ethnobiological information was collected using the multiple free listing ethnographic tool [37-38], which included questions focused on documenting which animals, fungi and plants they knew, the common or local name assigned to them in Spanish and Náhuatl, what use they had, and to be used, what part of the specimen was used.

The biological material was processed and identified depending on the taxonomic group, in general there were: a) specimen collections for fungi and plants, b) specimens or parts of animals donated by informants, c) specimen photographs, d) visual stimuli (use of field 
guides or posters with recognized species [34], e) association of traditional nomenclature with ethnobiological information in the area and f) published regional taxonomic lists. Species identification was carried out to the lowest possible taxonomic level, based on literature: fungi [39-40], plants [41-44], crustaceans [45], mollusks [46], diplopods [47] and vertebrates (fish [48-49]; amphibians and reptiles [50]; birds [51-53]; and mammals [54]).

\subsection{Data analysis}

The data were ordered in databases (spreadsheets of Excel) for each biological groups separately. Crustaceans and molluscs were included in the fish database as they were considered river fauna; the other biological groups are natural. The information in each database included: species, traditional names (Spanish/Náhuatl), categories of use and parts used. Ordinal matrices independent of the databases were too designed for each of the biological groups [55].

\subsubsection{BKSI: Biocultural Key Species Index}

This index is designed based on other methodological proposals [9-13]. It is made up of five subscripts that are: a) $\mathrm{MF}_{\mathrm{R}-\mathrm{A}}=$ Mention Frequency by Rank-Abundance, $\mathrm{AMO}=$ Average Order Mention, RTN= Richness Names Traditional, VUT/PU= Value Total Uses and Parts Used, and $\mathrm{O}=$ Species Origin; adding the correction factor (CF) of Amorozo and Gély [56] to each subscript in which the relation is included: informants-ethnographic sample $(\mathrm{n} / \mathrm{N})$.

$\mathrm{BKSI}=\sum\left(\mathbf{M F}_{\mathbf{R}-\mathbf{A}}+\mathbf{A M O}+\mathbf{R N T}+\mathbf{V T U} / \mathbf{P U}\right) \pm \mathbf{0}$

Where:

$\mathbf{M F}_{\mathbf{R}-\mathrm{A}}=$ Mention Frequency by Rank-Abundance

AMO= Average Mention Order

$\mathbf{R N T}=$ Richness Names Traditional

VTU/PU = Value Total Uses and Parts Used

$\mathbf{O}=+\mathbf{1}$ (for native species); $\mathbf{- 1}$ (for introduced specie)

\subsubsection{Mention Frequency by Rank-Abundance}


It expresses the positive responses given for an $i$ species in a sample of informants like Pieroni [12] or Garibay-Orijel et al. [13]. However, the frequency, that is, the possibility of finding a resource in daily life, may reflect its abundance in an ecosystem [9]; maintaining the relationship between abundant and rare species. Being necessary to evaluate this relationship, represented by $\mathrm{p}_{i}=$ proportional abundance of species $i$, that is, the number of individuals of $i$ species divided by the total number of individuals in the sample, which is known as the adjusted sample size [57].

$\mathbf{M F}_{\mathbf{R}-\mathbf{A}}=\mathbf{p i} * \operatorname{lnpi} * \mathbf{1 0}$

Where:

$\mathbf{p}_{i}=\left(\mathrm{f} / \sum \mathrm{f}\right)$

$\mathbf{f}=i$ species frequency; $\sum \mathrm{f}=$ summation of frequencies in the sample

\subsubsection{Average Mention Order}

It is obtained starting from the organization of the data in ordinal matrices proposed by Ryan et al. [55], where it is assumed that the mentioned species is random in a sample and all are represented in the sample (at least once); it acquires values close to zero when the mentions of species $i$ are few or one, therefore, the higher the value obtained, the greater proportion of representation of the $i$ species in the sample. Their analysis is based on value assignation to the ordering of the mentions for all species.

$\mathbf{O M P}=\frac{\text { Osp }_{i}}{\sum 0 s p_{\mathrm{n}}} * \mathbf{F C} * 100$

Where:

$\mathbf{O s p}_{i}=$ value assigned to positions for the $i$ species

$\operatorname{Osp}_{\mathbf{n}}=$ sum of rankings in the sample

$\mathbf{C F}=$ correction factor, that is, the proportion between the number of informants who mention $i$ species over the total number of informants $(\mathrm{n} / \mathrm{N})$

\subsubsection{Richness Names Traditional}


Following the basic principles of traditional classification [1,2] the richness of traditional names was estimated from the number of names assigned to an $i$ species, considering the greatest number of linguistic variants. First, the nomenclatural relationship (NR), was determined under the concepts of equity, proportionality, synonymy and homonymy; these last two mentioned by Freire and Pauly [58]. Equity is understood as the assignment of a traditional name to an $i$ species (1:1); proportionality as the association of more than one traditional name to more than one $i$ species $(>1:>1)$; synonymy is the ratio of more than one traditional name to an $i$ species $(>1: 1)$; and homonymy is equal to a traditional name associated with various taxa (1:>1; e.g. Table 1$)$.

On the other hand, based on the meaning of traditional names, as an indication of their cultural relevance, the value of linguistic interpretation (VIL) was obtained. Determining the nomenclatural category to which the name refers according to its meaning, these categories were assigned an arbitrary value. In general, four nomenclatural categories were identified, therefore, the value of linguistic interpretation depends on the number of categories identified, which ranges from 0.25 to 1 (Table 2).

$\mathbf{R N T}=\left(\frac{\mathbf{N t s p}_{\boldsymbol{i}}}{\sum \mathbf{N T}}\right) *(\mathbf{R N}+\mathbf{V I L}) *(\mathbf{F C}) * \mathbf{1 0}$

Where:

$\mathbf{N t s p}_{i}=$ traditional names assigned to the $i$ species

$\mathbf{N T}=$ sum of traditional names

$\mathbf{R N}=$ nomenclatural relationship

$\mathbf{V I L}=$ linguistic interpretation

$\mathbf{F C}=\mathrm{n} / \mathrm{N}$

Table 1. Nomenclatural relationships and examples with their assigned values.

\begin{tabular}{lllcc}
\hline $\begin{array}{c}\text { Nomenclatural } \\
\text { Relation }\end{array}$ & \multicolumn{1}{c}{$\begin{array}{c}\text { Tradicional Names } \\
\text { (Spanish/Náhuatl) }\end{array}$} & \multicolumn{1}{c}{ Specie } & $\begin{array}{c}\text { Nomenclatural } \\
\text { Relation }\end{array}$ & Value \\
\hline Equity & $\mathbf{1}$ & $\mathbf{1}$ & $\mathbf{1 : 1}$ & 1 \\
& Anona & Rollinia membranacea & $1 / 1$ & 1 \\
\hline Proportionality & $\mathbf{1}$ & $>\mathbf{1}$ & $>\mathbf{1 : > 1}$ & \\
& $\begin{array}{l}\text { orejita / orejita de viejita / } \\
\text { nalga de la abuelita / }\end{array}$ & $\begin{array}{l}\text { Auricularia auricula } \\
\text { Auricularia delicata }\end{array}$ & $6 / 3$ & 2 \\
\hline
\end{tabular}




\begin{tabular}{|c|c|c|c|c|}
\hline & $\begin{array}{l}\text { cuapetachiquinte / } \\
\text { cualeleshtli / chocoyos }\end{array}$ & $\begin{array}{l}\text { Auricularia } \\
\text { fuscosuccinea }\end{array}$ & & \\
\hline \multirow[t]{2}{*}{ Synonymy } & & 1 & $>1: 1$ & \\
\hline & $\begin{array}{l}\text { pájaro del cerro / pavo real } \\
\text { / tepetltototl }\end{array}$ & Crax rubra & $5 / 1$ & 5 \\
\hline \multirow[t]{7}{*}{ Homonymy } & 1 & $>1$ & $1:>1$ & \\
\hline & & Diona ipni & & \\
\hline & & Diona rasconis & & \\
\hline & & Gambusia vittata & $1 / 6$ & 0.16 \\
\hline & poxta & Xiphophorus continens & $1 / 0$ & 0.10 \\
\hline & & Xiphophorus malinche & & \\
\hline & & Xiphophorus variatus & & \\
\hline
\end{tabular}

Table 2. Nomenclatural categories, associated characters and examples for each category.

\begin{tabular}{|c|c|c|}
\hline Category & Type of character & $\begin{array}{c}\text { Value of } \\
\text { assignation }\end{array}$ \\
\hline Morphological & $\begin{array}{l}\text { shape, color, size, texture, association or similarity with } \\
\text { the morphology of another species } \\
\text { Cantharellus cibarius; xochilnanacatl (xochil=flower, } \\
\text { nanacatl=mushroom: flower-shaped mushroom) }\end{array}$ & 0.25 \\
\hline Behavior & $\begin{array}{l}\text { behavioral, sounds } \\
\text { Momotus momota; motmot (sound emitted by the bird) }\end{array}$ & 0.25 \\
\hline Ecologicall & $\begin{array}{l}\text { habits, ecosystem association, seasonality } \\
\text { Eira barbara; tepetlchichi (tepetl=hill, chichi=dog: dog } \\
\text { of the hill) }\end{array}$ & 0.25 \\
\hline Cultural & $\begin{array}{l}\text { reverential, association or likeness to an object or some } \\
\text { human aspect, types of uses } \\
\text { Euphorbia pulcherrima; tonantzinxihuitl } \\
\text { (tonantzin=virgin, xihuitl=flower: flower of the virgin) }\end{array}$ & 0.25 \\
\hline
\end{tabular}

\subsubsection{Value Total Uses and Parts Used}

Based on the different indices to evaluate the use value $[10,11,14,59,60]$, an index was integrated that includes the use value for each $i$ species, associated with the number of parts used for each $i$ species. The number of the categories depends on the biological group, as well as the number of used parts; so, there are different values for animals (mammals, birds, reptiles, amphibians, fish), fungi and plants.

$\mathbf{V T U} / \mathbf{P E}=\left(\mathbf{U s p}_{\boldsymbol{i}} / \sum \mathbf{U}\right) *\left(\mathbf{P s p}_{\boldsymbol{i}} / \sum \mathbf{P}\right) *(\mathbf{F C}) \times \mathbf{1 0}$

Where: 
$\mathbf{U s p}_{i}=$ use categories for the $i$ species

$\mathbf{U}=$ categories identified

$\mathbf{P s p}_{i}=$ used parts of the $i$ species

$\mathbf{P}=$ total parts used

$\mathbf{F C}=\mathrm{n} / \mathrm{N}$

The BKSI was applied in a spreadsheet (Excel) for each of the list species by biological group: plants and fungi; mammals, birds, amphibians, reptiles, and fish. Later, to know the data redundancy, a multiple linear correlation was performed, using the BKSI value as the independent variable and the values of each sub-index as the dependent variable; As well as a cluster analysis using the Euclidean distances as a grouping measure, statistical analyzes were performed in Past version 3.5 [61]. Finally, a scale of degrees of biocultural relevance was weighted, since the values are very different depending on the biological groups and their attributes. First, a distribution graph was made, assigning the value of the BKSI on the $\mathrm{x}$-axis and on the $\mathrm{y}$ axis. and the number (descending) of the number of species/ethnospecies; The sample was divided into 10 equal parts, assigning four categories of biocultural relevance: a) Maximum Biocultural Relevance (MaxBCR, 1/10 parts of the sample), b) High Biocultural Relevance (HBR, 2/10 of the sample), c) Medium Biocultural Relevance (MBR 3/10 of the sample) and d) Low Biocultural Relevance (LBR, 4/10 of the sample).

\subsection{Results}

A total of 4,399 mentions of fauna, flora and mycobiota known and / or exploited by the Nahuas of the community of El Barco in the Huastec Hidalguense region, corresponding to 549 traditional names (Spanish/Náhuatl); with 335 species, 301 genera and 150 taxonomic families; organized in the following biological groups: plants and fungi; fish, reptiles, amphibians, birds and mammals.

Plants are the biological group with the highest number of species with 201, related to 209 ethnospecies and with 308 traditional names; while the smallest group are fungi with 10 species, 11 ethnospecies and 24 traditional names. In addition, plants present the highest mention frequency value to all biological groups with 1436 mentions, and fungi the lowest 
frequency $(n=171)$. While the group with intermediate values is that of mammals with 31 species, 34 ethnospecies, 68 traditional names and 664 mentions.

The species that presented the highest value for BKSI by biological group are: for mammals Odocoileus virginianus 'venado/mazatl' (BKSI=10.82); in birds Crax rubra 'faisán/paisana bird/cerro bird/peacock/tepetltototl' with a value of $\mathrm{BKSI}=10.34$; for reptiles the ethnospecies 'coral snake/tzicatena' made up of a complex of snake species (Lampropeltis triangulum, Tantilla rubra, Tropidodipsas sartorii and Micrurus diastema; BKSI=9.26). For fish, a complex consisting of two species, Ictalurus mexicanus and Pylodictis olivaris known by the traditional names of 'catfish/xolotl/miquecuani/xolomichi' (BKSI=12.18). While for the fungi another complex of species integrated by Cantharellus cibarius, $C$. lateritius and $C$. odoratus $(\mathrm{BKSI}=19.07)$ to which traditionally the traditional names of 'chipahuachiquinte/yellow chiquinte/xochilnanacatl' are assigned; and finally, for the Psidium guajava 'guayaba/chalchocotl' plants with an BKSI= 3.85 (Table 3).

Table 3. Diversity of species known and used by the local people of the El Barco, Lolotla, Hidalgo, Mexico (Huastec). The traditional nomenclature is reported in Náhuatl and Spanish. The values obtained in the sub-index and the total value of the Biocultural Key Species Index, are reported for each of the species. MFR-A: Mention Frequency by Rank-Abundance; AMO: Average Mention Order; RNT: Richness Names Traditional; VTU/PU: Value Total Uses/Parts Used; O: Species Origin; BKSI: Biocultural Key Species Index.

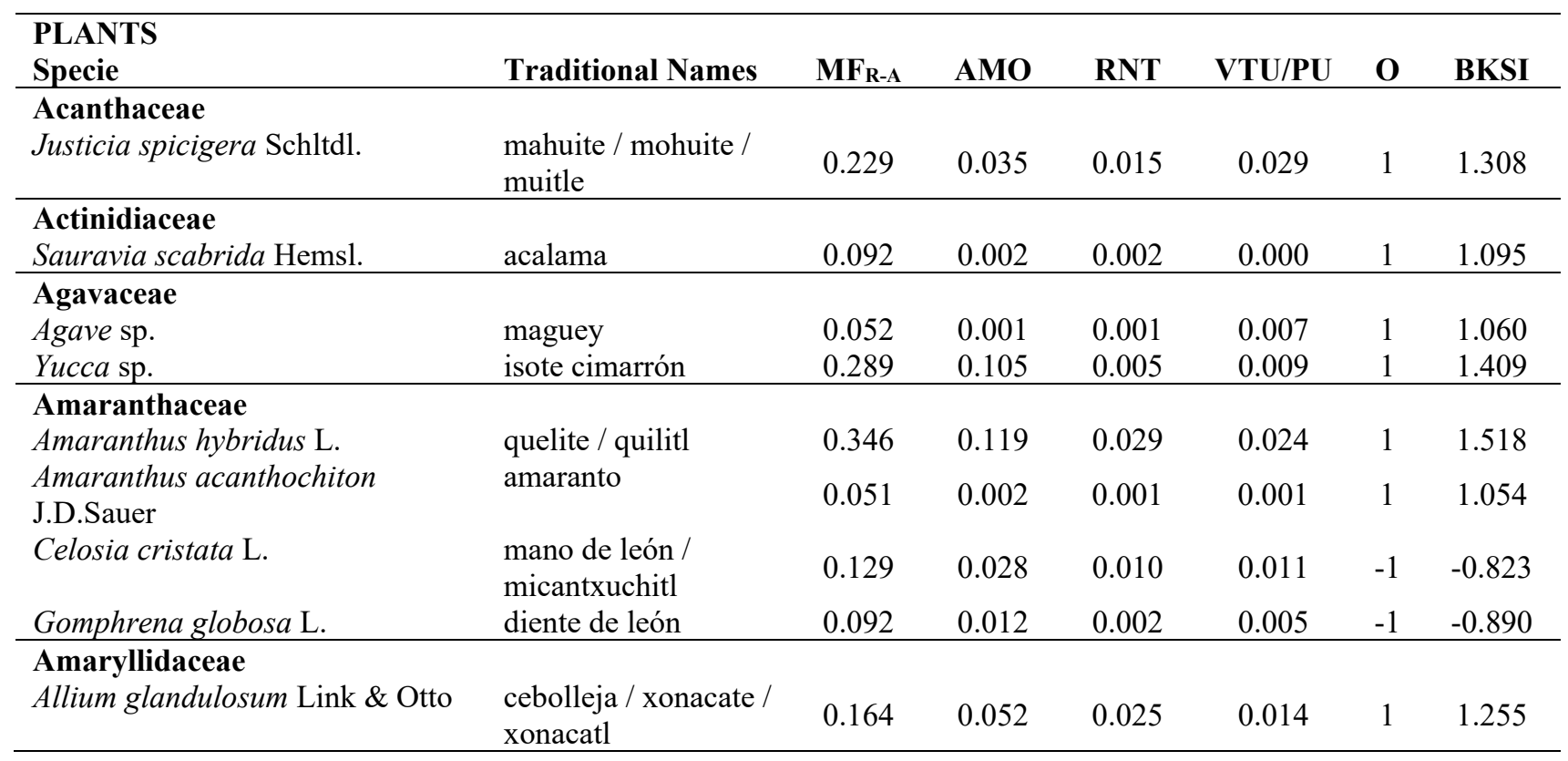




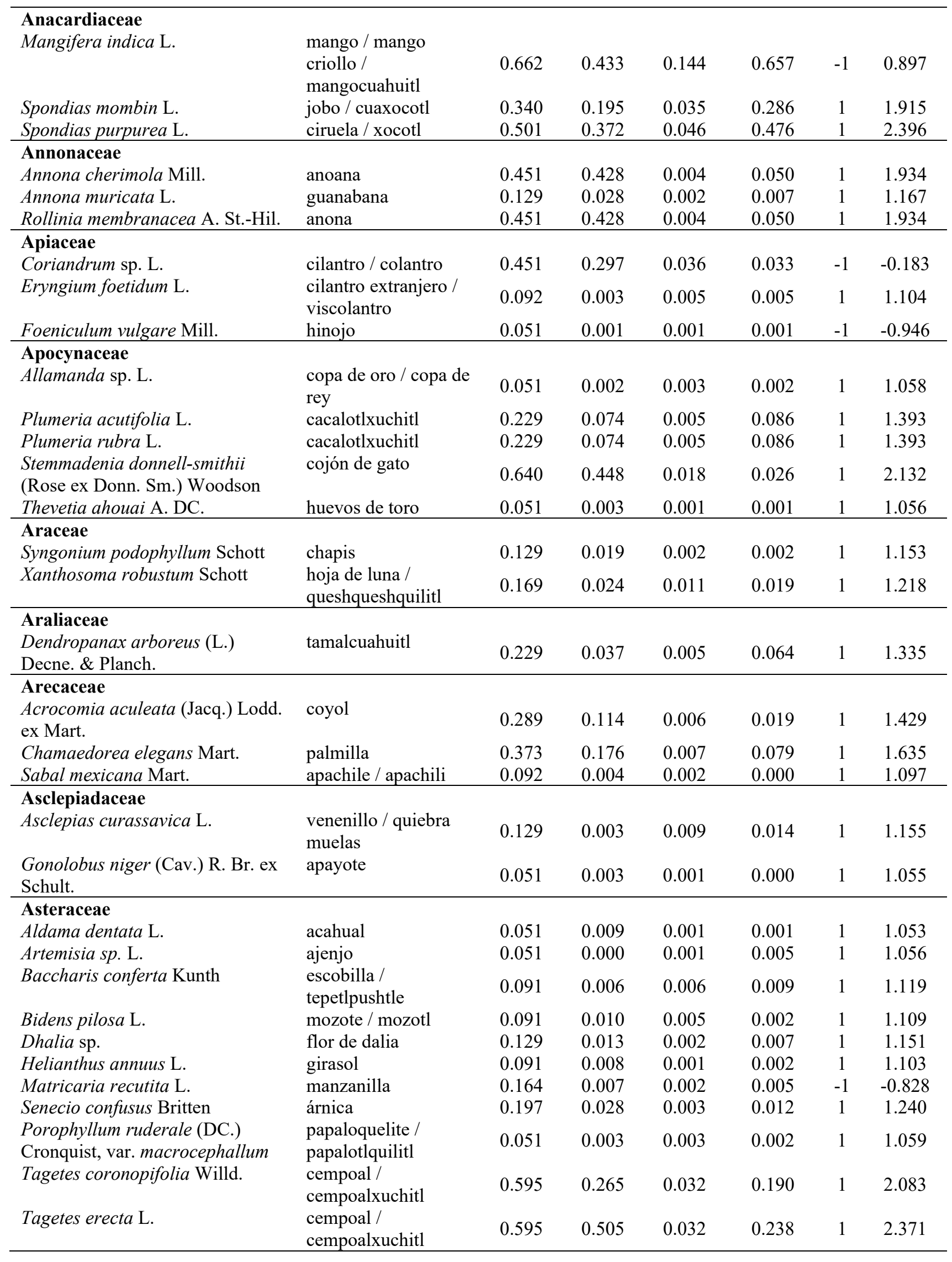




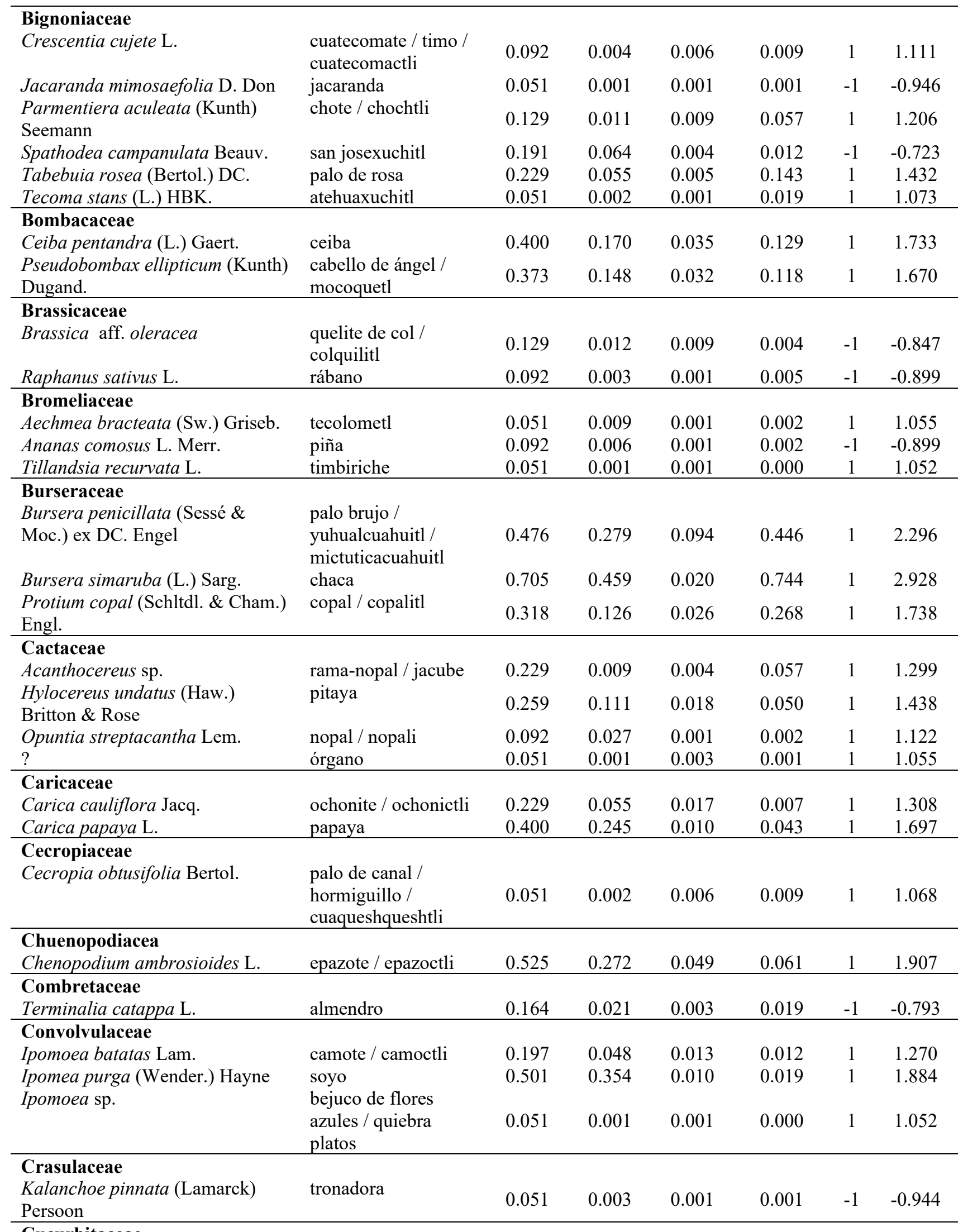

Cucurbitaceae 


\begin{tabular}{|c|c|c|c|c|c|c|c|}
\hline $\begin{array}{l}\text { Citrullus lanatus } \\
\text { (Thunb.) Matsum. \& Nakai }\end{array}$ & sandía & 0.051 & 0.002 & 0.001 & 0.001 & -1 & -0.946 \\
\hline Cucurbita maxima Lam. & calabaza & 0.259 & 0.086 & 0.004 & 0.025 & 1 & 1.375 \\
\hline $\begin{array}{l}\text { Sechium edule (Jacq.) Sw. } \\
\text { Chrysobalanaceae }\end{array}$ & chayote & 0.197 & 0.065 & 0.003 & 0.012 & 1 & 1.277 \\
\hline Couepia polyandra (Kunth) Rose & olopillo & 0.197 & 0.033 & 0.003 & 0.095 & 1 & 1.328 \\
\hline \multicolumn{8}{|l|}{ Cyperaceae } \\
\hline Cyperus odoratus L. & zacate & 0.051 & 0.002 & 0.001 & 0.001 & 1 & 1.054 \\
\hline \multicolumn{8}{|l|}{ Ebenaceae } \\
\hline $\begin{array}{l}\text { Diospyros digyna Jacq. Pl. hort. } \\
\text { schoenbr }\end{array}$ & zapote negro & 0.164 & 0.044 & 0.0032 & 0.029 & 1 & 1.240 \\
\hline \multicolumn{8}{|l|}{ Euphorbiaceae } \\
\hline $\begin{array}{l}\text { Cnidoscolus aconitifolius (Mill.) } \\
\text { I. M. Johnst. }\end{array}$ & $\begin{array}{l}\text { chaya / } \\
\text { chichacuahuitl }\end{array}$ & 0.051 & 0.001 & 0.003 & 0.001 & 1 & 1.056 \\
\hline $\begin{array}{l}\text { Cnidoscolus multilobus (Pax) } \\
\text { I.M. Johnst. }\end{array}$ & tesonquilitl & 0.051 & 0.003 & 0.001 & 0.000 & 1 & 1.054 \\
\hline Croton draco Schltdl. & $\begin{array}{l}\text { sangregado / palo de } \\
\text { sangre / escuahuitl }\end{array}$ & 0.092 & 0.008 & 0.012 & 0.014 & 1 & 1.126 \\
\hline Croton pulcher Müll. Arg. & soliman & 0.129 & 0.011 & 0.002 & 0.000 & 1 & 1.142 \\
\hline $\begin{array}{l}\text { Euphorbia pulcherrima Willd. ex } \\
\text { Klotzsch }\end{array}$ & $\begin{array}{l}\text { flor de la virgen / } \\
\text { noche buena / } \\
\text { tonantzinxiuhutl }\end{array}$ & 0.229 & 0.098 & 0.037 & 0.043 & 1 & 1.407 \\
\hline Jatropha curcas L. & piñón & 0.400 & 0.141 & 0.008 & 0.129 & 1 & 1.677 \\
\hline Manihot esculenta Crantz & yuca & 0.164 & 0.033 & 0.003 & 0.009 & 1 & 1.209 \\
\hline Ricinum communis L. & higuerilla & 0.092 & 0.004 & 0.001 & 0.002 & 1 & 1.099 \\
\hline \multicolumn{8}{|l|}{ Equitaceae } \\
\hline Equisetum sp. & $\begin{array}{l}\text { cola de caballo / } \\
\text { aquiztopile }\end{array}$ & 0.052 & 0.001 & 0.003 & 0.001 & 1 & 1.055 \\
\hline \multicolumn{8}{|l|}{ Fabaceae } \\
\hline Acacia cornigera L. Willd. & toro cuerno & 0.129 & 0.0170 & 0.002 & 0.021 & 1 & 1.170 \\
\hline $\begin{array}{l}\text { Acosmium panamense (Benth.) } \\
\text { Yakovlev }\end{array}$ & $\begin{array}{l}\text { hueso de tigre / } \\
\text { huesillo }\end{array}$ & 0.197 & 0.0471 & 0.014 & 0.036 & 1 & 1.294 \\
\hline Amicia zygomeris DC. & papaloxihuitl & 0.050 & 0.000 & 0.000 & 0.001 & 1 & 1.053 \\
\hline Arachis hypogaea $\mathrm{L}$. & cacahuate / cacahuatl & 0.129 & 0.003 & 0.009 & 0.007 & 1 & 1.147 \\
\hline Bauhinia divaricata $\mathrm{L}$. & $\begin{array}{l}\text { pata de buey / } \\
\text { zahuach }\end{array}$ & 0.197 & 0.035 & 0.014 & 0.006 & 1 & 1.252 \\
\hline Caesalpinia pulcherrima Benth. & $\begin{array}{l}\text { árbol de rosal / } \\
\text { tabachín }\end{array}$ & 0.092 & 0.006 & 0.006 & 0.002 & 1 & 1.106 \\
\hline Cajanus cajan (L.) Millsp. & lentejilla & 0.260 & 0.058 & 0.004 & 0.008 & -1 & -0.669 \\
\hline Cassia fistula $\mathrm{L}$. & lluvia de oro & 0.164 & 0.028 & 0.003 & 0.095 & 1 & 1.290 \\
\hline $\begin{array}{l}\text { Calliandra grandiflora (L' Hér.) } \\
\text { Benth. }\end{array}$ & $\begin{array}{l}\text { cabello de ángel / } \\
\text { tzotzocometl }\end{array}$ & 0.092 & 0.008 & 0.006 & 0.002 & 1 & 1.108 \\
\hline $\begin{array}{l}\text { Dalbergia palo-escrito Rzed. \& } \\
\text { Guridi-Gómez }\end{array}$ & $\begin{array}{l}\text { palo escrito / } \\
\text { tlacuilocuahuitl }\end{array}$ & 0.451 & 0.172 & 0.040 & 0.250 & 1 & 1.913 \\
\hline Delonix regia (Bojer) Raf. & $\begin{array}{l}\text { framboyán / flor de } \\
\text { mayo / flor de las } \\
\text { madres }\end{array}$ & 0.572 & 0.588 & 0.207 & 0.362 & -1 & 0.729 \\
\hline Diphysa senoides Benth. & $\begin{array}{l}\text { quiebra hacha / } \\
\text { quebrache / } \\
\text { quebrachi }\end{array}$ & 0.129 & 0.012 & 0.009 & 0.014 & 1 & 1.164 \\
\hline Erythrina americana Mill. & $\begin{array}{l}\text { pemuche / colorín / } \\
\text { pemuch }\end{array}$ & 0.595 & 0.519 & 0.125 & 0.714 & 1 & 2.953 \\
\hline Erythrina lanata Rose & pemuche rosado & 0.129 & 0.012 & 0.002 & 0.0215 & 1 & 1.165 \\
\hline $\begin{array}{l}\text { Eysenhardtia platycarpa Pennell } \\
\text { \& Saff. }\end{array}$ & palo azul & 0.259 & 0.095 & 0.006 & 0.0668 & 1 & 1.426 \\
\hline Cliricidia sepium (Jacq.) Steud. & cacahuitl & 0.051 & 0.000 & 0.000 & 0.014 & 1 & 1.066 \\
\hline
\end{tabular}


Leucaena leucocephala (Lam.) de árbol de guaje /

Wit

Mucuna pruriens (L.) DC.

guactli

Pachyrrhizus erosus (L.) Urb.

Phaseolus coccineus L.

Phaseolus sp.

Piscidia piscipula (L.) Sarg.

Pithecellobium dulce (Roxb.)

Benth.

Senna occidentalis (L.) Irwin

Tamarindus indica $\mathrm{L}$.

Trifolium sp.

pica pica

0.129

0.016

0.008

0.043

1

1.196

jícama

0.051

0.001

0.001

0.000

$\begin{array}{ll}1 & 1.053 \\ 1 & 1.248\end{array}$

frijol

$0.197 \quad 0.035$

0.003

0.012

cashtilán

0.197

0.079

0.003

0.012

1.291

chijol

$0.197 \quad 0.041$

0.003

0.012

1.253

frijolillo / humos

$0.057 \quad 0.000$

0.001

0.002

1.054

cafecillo

0.426

0.347

0.037

0.186

1.996

tamarindo /

tamarindocuahuitl

$0.346 \quad 0.108$

0.006

0.000

1.460

quelite estrella

0.525

0.381

0.049

0.486

0.441

Vicia faba L.

haba

$0.129 \quad 0.010$

0.002

0.004

1.145

0.092

0.002

0.001

0.002 $-0.903$

\section{Fagaceae}

Quercus oleoides Schiltdl. \&

encino / encino

Cham.

blanco / tiocuahuitl /

0.373

0.162

0.127

0.262

1.924

itztactiocuahuitl

\section{Gramineae}

Lasiacis divaricata (L) A. S. bambú

Hitch.

0.197

0.020

0.003

0.012

1

1.233

\begin{tabular}{|c|c|c|c|c|c|c|c|}
\hline $\begin{array}{l}\text { Hamemelidaceae } \\
\text { Liquidambar macrophylla Oerst. }\end{array}$ & suchiate / suchiatl & 0.229 & 0.053 & 0.017 & 0.064 & 1 & 1.363 \\
\hline $\begin{array}{l}\text { Heliconiaceae } \\
\text { Heliconia schiedeana Klotzch }\end{array}$ & papatla / papatl & 0.164 & 0.009 & 0.011 & 0.038 & 1 & 1.222 \\
\hline $\begin{array}{l}\text { Lamiaceae } \\
\text { Clerodendrum thomsoniae Balf. } \\
\text { Mentha spicata } \mathrm{L} . \\
\text { Ocimum basilicum } \mathrm{L} . \\
\text { Origanum mejorana } \mathrm{L} . \\
\text { Origanum vulgare } \mathrm{L} . \\
\text { Rosmarinus officinalis } \mathrm{L} . \\
\text { Sesamum indicum } \mathrm{L} \text {. } \\
\end{array}$ & $\begin{array}{l}\text { velo de novia } \\
\text { hierba buena } \\
\text { albahaca } \\
\text { mejorana } \\
\text { orégano } \\
\text { romero } \\
\text { ajonjolí }\end{array}$ & $\begin{array}{l}0.092 \\
0.501 \\
0.400 \\
0.051 \\
0.051 \\
0.051 \\
0.129 \\
\end{array}$ & $\begin{array}{l}0.007 \\
0.247 \\
0.102 \\
0.002 \\
0.001 \\
0.001 \\
0.007 \\
\end{array}$ & $\begin{array}{l}0.001 \\
0.010 \\
0.008 \\
0.001 \\
0.001 \\
0.001 \\
0.002 \\
\end{array}$ & $\begin{array}{l}0.009 \\
0.057 \\
0.086 \\
0.001 \\
0.004 \\
0.005 \\
0.007 \\
\end{array}$ & $\begin{array}{l}-1 \\
-1 \\
-1 \\
-1 \\
-1 \\
-1 \\
-1\end{array}$ & $\begin{array}{l}-0.895 \\
-0.188 \\
-0.408 \\
-0.945 \\
-0.944 \\
-0.943 \\
-0.855 \\
\end{array}$ \\
\hline $\begin{array}{l}\text { Lauraceae } \\
\text { Cinnamomum camphora L. } \\
\text { Cinnamomum verum J. Presl } \\
\text { Licaria capitata (Cham. \& } \\
\text { Schdtl.) Kosterm. } \\
\text { Persea americana Miller } \\
\text { Persea americana var. drymifolia } \\
\text { (Schldl. \& Cham.) S.F. Blake } \\
\text { Persea schiedeana } \text { Nees }\end{array}$ & $\begin{array}{l}\text { alcanfor } \\
\text { canela } \\
\text { xolime } \\
\text { aguacate / aguacatl } \\
\text { aguacate oloroso / } \\
\text { aguacate tlapanilli } \\
\text { pahua / pahuatl }\end{array}$ & $\begin{array}{r}0.129 \\
0.129 \\
0.051 \\
0.6622 \\
0.2289 \\
0.346\end{array}$ & $\begin{array}{r}0.019 \\
0.018 \\
0.001 \\
0.7136 \\
0.0771 \\
0.213\end{array}$ & $\begin{array}{c}0.002 \\
0.002 \\
0.001 \\
0.0663 \\
0.0173 \\
0.029\end{array}$ & $\begin{array}{c}0.000 \\
0.011 \\
0.001 \\
0.6845 \\
0.0429 \\
0.071 \\
\end{array}$ & $\begin{array}{l}-1 \\
-1 \\
1 \\
1 \\
1 \\
1\end{array}$ & $\begin{array}{c}-0.850 \\
-0.840 \\
1.054 \\
3.127 \\
1.366 \\
1.660\end{array}$ \\
\hline $\begin{array}{l}\text { Liliaceae } \\
\text { Allium sativum } \text { L. } \\
\text { Loranthaceae } \\
\text { Struthanthus crassipes (Oliver) } \\
\text { Eichler } \\
\text { Maonoliacego }\end{array}$ & $\begin{array}{l}\text { ajo } \\
\text { seca palo }\end{array}$ & 0.092 & 0.005 & 0.001 & 0.000 & -1 & 1.098 \\
\hline $\begin{array}{l}\text { Magnoliaceae } \\
\text { Magnolia grandifolia } \text { L. } \\
\text { Talauma mexicana } \text { (DC.) Don }\end{array}$ & $\begin{array}{l}\text { magnolia } \\
\text { yoloxuchitl }\end{array}$ & $\begin{array}{l}0.129 \\
0.259 \\
\end{array}$ & $\begin{array}{l}0.009 \\
0.068\end{array}$ & $\begin{array}{l}0.002 \\
0.006 \\
\end{array}$ & $\begin{array}{l}0.007 \\
0.100\end{array}$ & $\begin{array}{c}-1 \\
1 \\
\end{array}$ & $\begin{array}{c}-0.853 \\
1.433 \\
\end{array}$ \\
\hline $\begin{array}{l}\text { Malpighiaceae } \\
\text { Bunchosia palmeri S. Watson } \\
\text { Byrsonima crassifolia } \text { (L.) Kunth }\end{array}$ & $\begin{array}{l}\text { huasha } \\
\text { nantzi }\end{array}$ & $\begin{array}{l}0.164 \\
0.129 \\
\end{array}$ & $\begin{array}{l}0.016 \\
0.009\end{array}$ & $\begin{array}{l}0.003 \\
0.002\end{array}$ & $\begin{array}{l}0.005 \\
0.032\end{array}$ & $\begin{array}{l}1 \\
1\end{array}$ & $\begin{array}{l}1.187 \\
1.173\end{array}$ \\
\hline $\begin{array}{l}\text { Malvaceae } \\
\text { Hibiscus rosa-sinensis L. } \\
\text { Hibiscus sabdariffa L. }\end{array}$ & $\begin{array}{l}\text { tulipán } \\
\text { jamaica }\end{array}$ & $\begin{array}{l}0.164 \\
0.092\end{array}$ & $\begin{array}{l}0.045 \\
0.007\end{array}$ & $\begin{array}{l}0.003 \\
0.001\end{array}$ & $\begin{array}{l}0.019 \\
0.005\end{array}$ & -1 & $\begin{array}{c}1.230 \\
-0.895\end{array}$ \\
\hline
\end{tabular}




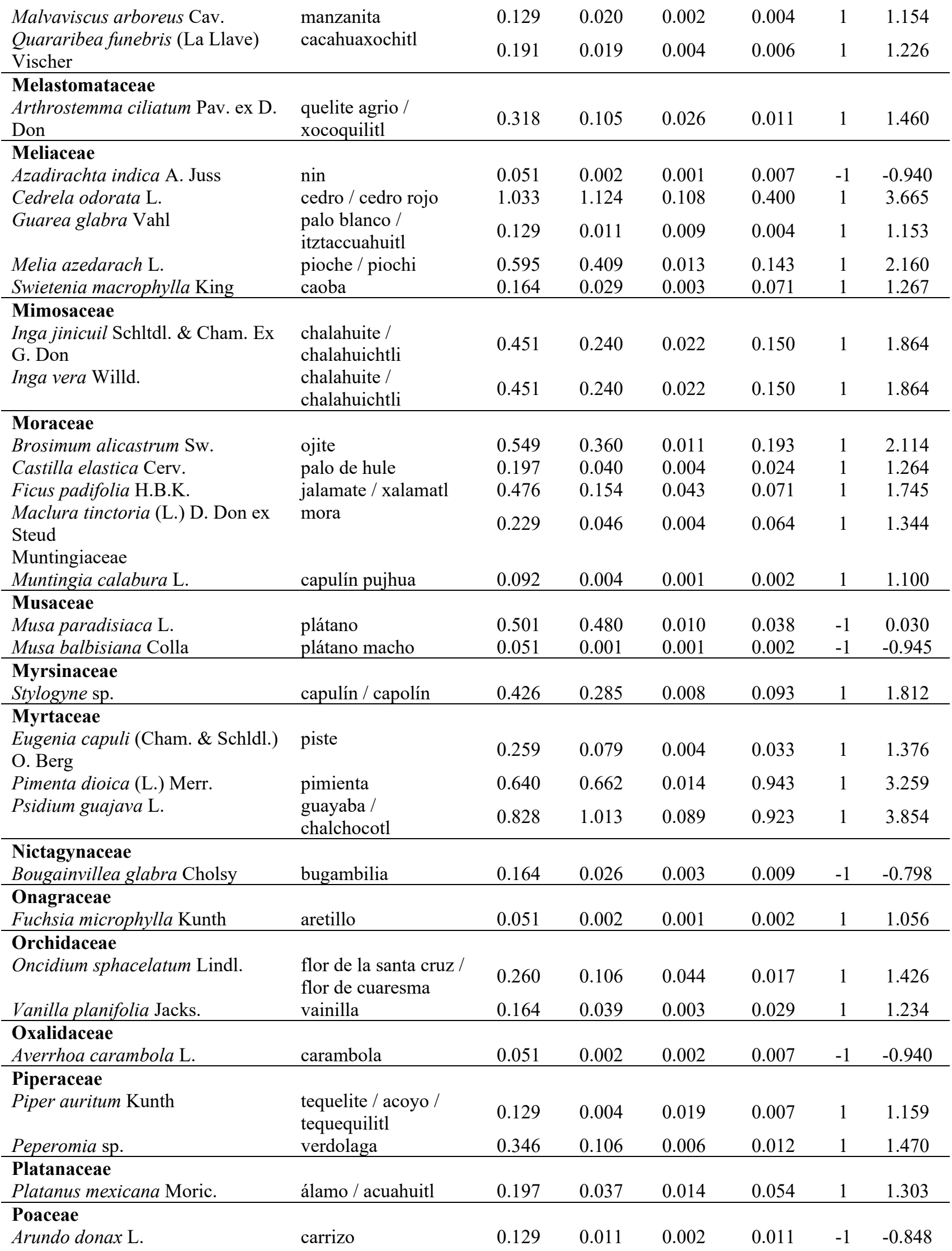




\begin{tabular}{|c|c|c|c|c|c|c|c|}
\hline Echinochloa colona (L.) Link & zacate colorado & 0.092 & 0.004 & 0.001 & 0.002 & -1 & -0.901 \\
\hline Digitaria decumbens Stent. & pasto pamgola & 0.051 & 0.002 & 0.001 & 0.001 & -1 & -0.945 \\
\hline Guadua amplexifolia J.Presl & otate & 0.197 & 0.038 & 0.003 & 0.012 & 1 & 1.250 \\
\hline $\begin{array}{l}\text { Pennisetum purpureum } \\
\text { Schumach. }\end{array}$ & pasto taiwán & 0.051 & 0.002 & 0.001 & 0.001 & -1 & -0.946 \\
\hline Saccharum officinarum L. & caña de azúcar & 0.229 & 0.071 & 0.005 & 0.043 & -1 & -0.652 \\
\hline Zea mays L. & maíz & 0.051 & 0.001 & 0.001 & 0.007 & 1 & 1.060 \\
\hline \multicolumn{8}{|l|}{ Polygonaceae } \\
\hline Rumex sp. & lengua de vaca & 0.092 & 0.003 & 0.002 & 0.009 & 1 & 1.106 \\
\hline \multicolumn{8}{|l|}{ Pteridaceae } \\
\hline Adiantum sp. & pesmita & 0.092 & 0.005 & 0.001 & 0.000 & 1 & 1.097 \\
\hline Pteridaceae & & & & & & & \\
\hline $\begin{array}{l}\text { Tectaria heracleifolia (Willd.) } \\
\text { Underw. }\end{array}$ & pesmita & 0.092 & 0.005 & 0.001 & 0.002 & 1 & 1.100 \\
\hline \multicolumn{8}{|l|}{ Rhamnaceae } \\
\hline $\begin{array}{l}\text { Karwinskia humboldtiana } \\
\text { (Roemer \& Schultes) Zucc. }\end{array}$ & $\begin{array}{l}\text { capulín de zorra / } \\
\text { capulín cimarrón }\end{array}$ & 0.164 & 0.037 & 0.011 & 0.019 & 1 & 1.231 \\
\hline \multicolumn{8}{|l|}{ Rosaceae } \\
\hline Prunus persica (L.) Batsch. & durazno & 0.197 & 0.046 & 0.003 & 0.012 & -1 & -0.741 \\
\hline \multicolumn{8}{|l|}{ Rubiaceae } \\
\hline Coffea arabica $\mathrm{L}$. & & 0.549 & 0.539 & 0.011 & 0.043 & -1 & 0.142 \\
\hline Hamelia patens Jacq. & $\begin{array}{l}\text { maduro zapote / } \\
\text { maduro plantor }\end{array}$ & 0.501 & 0.185 & 0.041 & 0.000 & 1 & 1.728 \\
\hline \multicolumn{8}{|l|}{ Rutaceae } \\
\hline Casimiroa edulis Llave \& Lex. & $\begin{array}{l}\text { zapote blanco / } \\
\text { iztaczapotl }\end{array}$ & 0.051 & 0.002 & 0.003 & 0.014 & 1 & 1.070 \\
\hline $\begin{array}{l}\text { Citrus aurantifolia (Christm.) } \\
\text { Swingle }\end{array}$ & $\begin{array}{l}\text { naranja cimarrona / } \\
\text { naranja agría }\end{array}$ & 0.164 & 0.042 & 0.010 & 0.005 & -1 & -0.780 \\
\hline Citrus x sinensis Osbeck & $\begin{array}{l}\text { naranja / } \\
\text { naranjacuahuitl }\end{array}$ & 0.726 & 0.633 & 0.075 & 0.062 & -1 & 0.496 \\
\hline Citrus limetta Risso & lima / limacuahuitl & 0.260 & 0.138 & 0.020 & 0.017 & -1 & -0.565 \\
\hline $\begin{array}{l}\text { Citrus maxima (Burm. ex } \\
\text { Rumph.) Merr. }\end{array}$ & $\begin{array}{l}\text { limón / } \\
\text { lemonjcuahuitl }\end{array}$ & 0.618 & 0.778 & 0.061 & 0.075 & -1 & 0.531 \\
\hline Citrus reticulata Blanco & $\begin{array}{l}\text { mandarina / } \\
\text { mandarinacuahuitl }\end{array}$ & 0.476 & 0.273 & 0.043 & 0.054 & -1 & -0.154 \\
\hline Citrus x paradisi Macfad. & $\begin{array}{l}\text { toronja / } \\
\text { tojonjacuahuitl }\end{array}$ & 0.476 & 0.198 & 0.043 & 0.038 & -1 & -0.247 \\
\hline $\begin{array}{l}\text { Murraya paniculata Jokoening Ex } \\
\text { L. }\end{array}$ & limonaria & 0.229 & 0.067 & 0.004 & 0.007 & -1 & -0.694 \\
\hline Ruta chalepensis L. & ruda & 0.051 & 0.000 & 0.001 & 0.002 & -1 & -0.946 \\
\hline \multicolumn{8}{|l|}{ Salicacaea } \\
\hline Salix humboldtiana Willd. & sauce & 0.259 & 0.086 & 0.004 & 0.008 & 1 & 1.358 \\
\hline $\begin{array}{l}\text { Zuelania guidonia (Sw.) Britt. \& } \\
\text { Millsp. }\end{array}$ & volarín & 0.229 & 0.054 & 0.004 & 0.007 & 1 & 1.294 \\
\hline \multicolumn{8}{|l|}{ Sapindaceae } \\
\hline Litchi chinensis Sonn. & lichi / lichicuahuitl & 0.197 & 0.057 & 0.014 & 0.036 & -1 & -0.695 \\
\hline Sapindus saponaria $\mathrm{L}$. & jaboncito & 0.197 & 0.035 & 0.004 & 0.012 & 1 & 1.248 \\
\hline \multicolumn{8}{|l|}{ Sapotaceae } \\
\hline Manilkara zapota (L.) van Royen & $\begin{array}{l}\text { chicozapote / } \\
\text { xicotlzapotl }\end{array}$ & 0.525 & 0.441 & 0.049 & 0.243 & 1 & 2.258 \\
\hline $\begin{array}{l}\text { Pouteria campechiana (Kunth) } \\
\text { Baehni }\end{array}$ & zapote amarillo & 0.197 & 0.044 & 0.004 & 0.075 & 1 & 1.317 \\
\hline $\begin{array}{l}\text { Pouteria sapota (Jacq.) H.E. } \\
\text { Moore \& Stearn. }\end{array}$ & $\begin{array}{l}\text { zapote mamey / } \\
\text { zapotl }\end{array}$ & 0.662 & 0.796 & 0.015 & 0.548 & 1 & 3.021 \\
\hline $\begin{array}{l}\text { Simaroubaceae } \\
\text { Simarouba glauca DC. }\end{array}$ & pistache & 0.340 & 0.159 & 0.008 & 0.171 & 1 & 1.738 \\
\hline
\end{tabular}




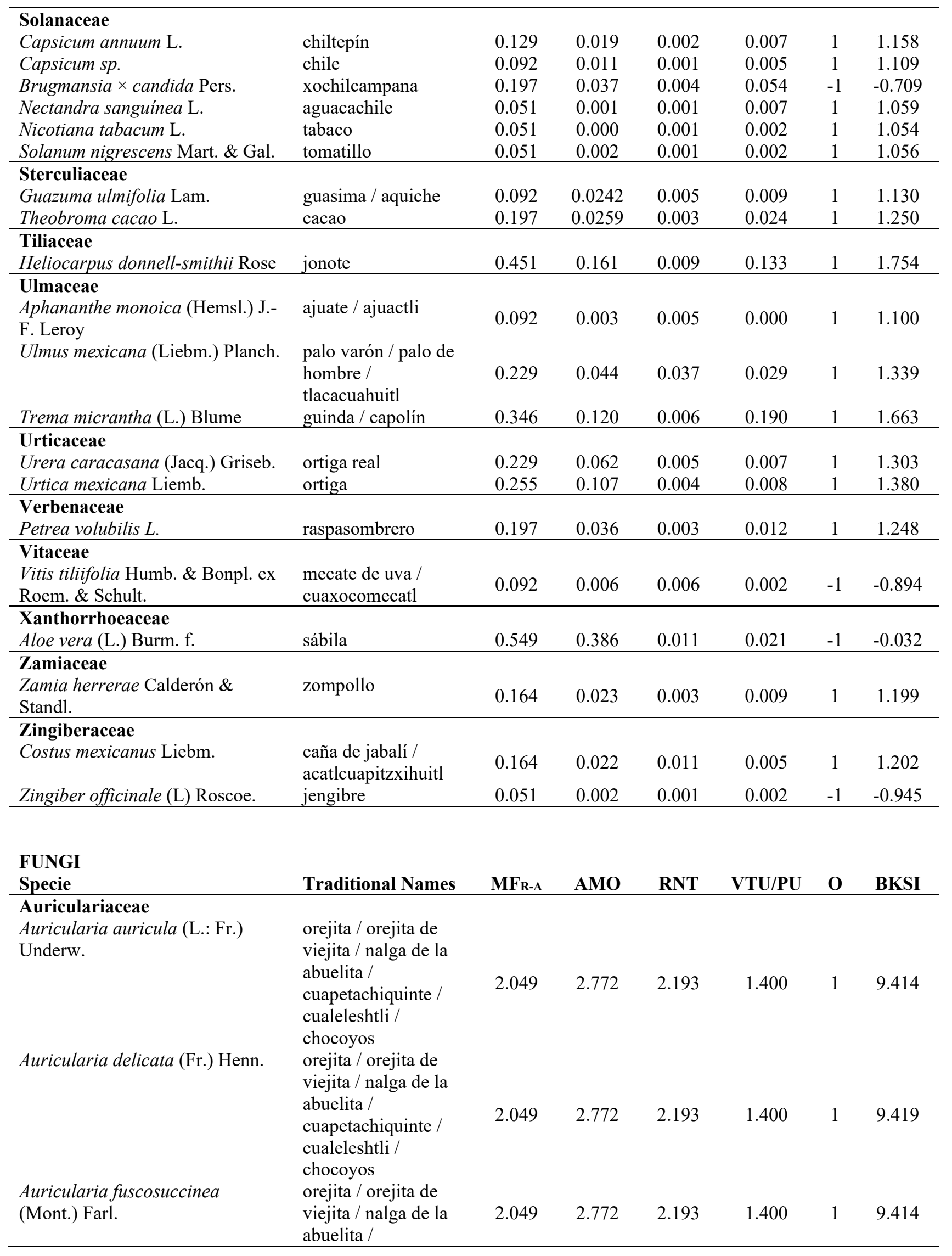


cuapetachiquinte /

cualeleshtli /

chocoyos

\begin{tabular}{|c|c|c|c|c|c|c|c|}
\hline \multicolumn{8}{|l|}{ Pleurotaceae } \\
\hline Pleurotus djamour (Fr.) Boedj. & $\begin{array}{l}\text { totomoshchiquinte / } \\
\text { totomoshnanacatl }\end{array}$ & 2.370 & 1.919 & 0.600 & 3.600 & 1 & 9.489 \\
\hline Pleurotu albidus (Berk.) Pegler & $\begin{array}{l}\text { hongo patón blanco / } \\
\text { chiquinte blanco / } \\
\text { iztacnanacatl }\end{array}$ & 2.756 & 3.777 & 1.200 & 4.800 & 1 & 13.533 \\
\hline \multicolumn{8}{|l|}{ Schizophyllaceae } \\
\hline Schizophyllum commune Fr. & chiquinte gris & 0.709 & 0.107 & 0.050 & 0.300 & 1 & 2.166 \\
\hline \multicolumn{8}{|l|}{ Cantharellaceae } \\
\hline Cantharellus cibarius Fr. & $\begin{array}{l}\text { chipahuachiquinte / } \\
\text { amarillo-chiquinte / } \\
\text { xochilnanacatl }\end{array}$ & 2.864 & 8.711 & 1.300 & 5.200 & 1 & 19.075 \\
\hline $\begin{array}{l}\text { Cantharellus lateritius (Berk.) } \\
\text { Singer }\end{array}$ & $\begin{array}{l}\text { chipahuachiquinte / } \\
\text { amarillo-chiquinte / } \\
\text { xochilnanacatl }\end{array}$ & 2.864 & 8.711 & 1.300 & 5.200 & 1 & 19.075 \\
\hline $\begin{array}{l}\text { Cantharellus aff. odoratus } \\
\text { (Schw.) Fr. }\end{array}$ & $\begin{array}{l}\text { chipahuachiquinte / } \\
\text { amarillo-chiquinte / } \\
\text { xochilnanacatl }\end{array}$ & 2.864 & 8.711 & 1.300 & 5.200 & 1 & 19.075 \\
\hline Cantharellus sp. & $\begin{array}{l}\text { xochilnanacatl } \\
\text { moradito / moradito }\end{array}$ & 0.878 & 0.223 & 0.111 & 0.800 & 1 & 3.013 \\
\hline \multicolumn{8}{|l|}{ Ustilaginaceae } \\
\hline Ustilago maydis (D.C.) Corda & $\begin{array}{l}\text { hongo de milpa / } \\
\text { hongo de maíz / } \\
\text { cuitlacoche }\end{array}$ & 0.5203 & 0.0914 & 0.2333 & 0.200 & 1 & 2.045 \\
\hline
\end{tabular}

\begin{tabular}{|c|c|c|c|c|c|c|c|}
\hline $\begin{array}{l}\text { FISHES } \\
\text { Specie }\end{array}$ & Traditional Names & $M_{\text {R-A }}$ & AMO & RNT & VTU/PU & $\mathbf{O}$ & BKSI \\
\hline Gastropoda Cuvier, 1797 & caracoles & 0.459 & 0.192 & 0.048 & 0.140 & 1 & 1.839 \\
\hline $\begin{array}{l}\text { Cambaridae } \\
\text { Procambarus sp. }\end{array}$ & techichis / burritos & 1.550 & 5.302 & 1.148 & 1.480 & 1 & 10.480 \\
\hline $\begin{array}{l}\text { Palaemonidae } \\
\text { Macrobranchium sp. } \\
?\end{array}$ & $\begin{array}{l}\text { acamayas } \\
\text { cosoles }\end{array}$ & $\begin{array}{l}1.494 \\
1.809 \\
\end{array}$ & $\begin{array}{l}4.101 \\
6.546 \\
\end{array}$ & $\begin{array}{l}0.302 \\
0.405 \\
\end{array}$ & $\begin{array}{l}1.400 \\
1.880 \\
\end{array}$ & $\begin{array}{l}1 \\
1\end{array}$ & $\begin{array}{c}8.296 \\
11.639 \\
\end{array}$ \\
\hline $\begin{array}{l}\text { Angulidae } \\
\text { Anguilla rostrata Lesueur, } 1821\end{array}$ & coatlmichi & 0.605 & 0.516 & 0.310 & 2.000 & 1 & 4.431 \\
\hline $\begin{array}{l}\text { Lepisosteidae } \\
\text { Lepisosteus osseus Linnaeus, } \\
1758\end{array}$ & $\begin{array}{l}\text { pez aguja / } \\
\text { huitzilmichi }\end{array}$ & 0.821 & 1.024 & 0.465 & 1.800 & 1 & 5.111 \\
\hline $\begin{array}{l}\text { Calostomide } \\
\text { Ictiobus labiosus (Meek, 1904) }\end{array}$ & $\begin{array}{l}\text { trompa de puerco / } \\
\text { pitzolmichi }\end{array}$ & 0.605 & 0.355 & 0.310 & 0.400 & 1 & 2.670 \\
\hline $\begin{array}{l}\text { Characidae } \\
\text { Cichlasoma labridens (Pellegrin, } \\
\text { 1903) }\end{array}$ & $\begin{array}{l}\text { mojarras / tecoxitle/ } \\
\text { xomaquictle/ } \\
\text { xumaquictle }\end{array}$ & 1.709 & 2.562 & 1.210 & 2.580 & 1 & 9.061 \\
\hline $\begin{array}{l}\text { Herichthys tamasopoensis } \\
\text { (Artigas Azas, 1993) }\end{array}$ & $\begin{array}{l}\text { mojarras / tecoxitle/ } \\
\text { xomaquictle/ } \\
\text { xumaquictle }\end{array}$ & 1.709 & 2.562 & 1.210 & 2.580 & 1 & 9.061 \\
\hline $\begin{array}{l}\text { Cichlidae } \\
\text { Amatatlania nigrofasciata } \\
\text { (Günther, 1867) }\end{array}$ & $\begin{array}{l}\text { mojarras / tecoxitle/ } \\
\text { xomaquictle/ } \\
\text { xumaquictle }\end{array}$ & 1.709 & 2.562 & 1.201 & 2.580 & -1 & 7.061 \\
\hline
\end{tabular}




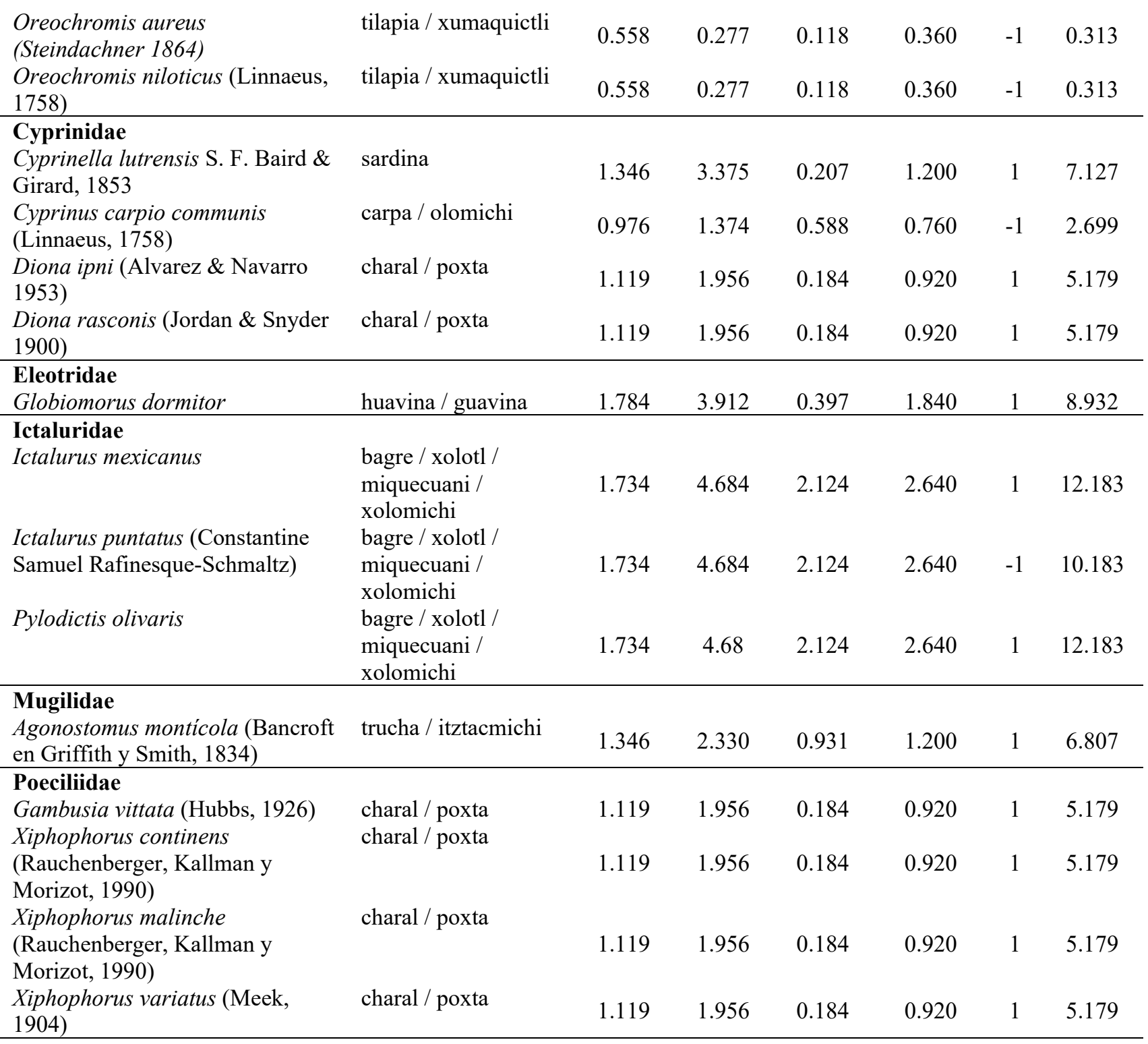

\begin{tabular}{|c|c|c|c|c|c|c|c|}
\hline $\begin{array}{l}\text { HERPETOFAUNAL } \\
\text { Specie }\end{array}$ & Traditional Names & $\mathbf{M F}_{\mathrm{R}-\mathrm{A}}$ & AMO & RNT & VTU/PU & $\mathbf{O}$ & BKSI \\
\hline Amphibia & ranitas & 1.177 & 1.189 & 0.118 & 0.076 & 1 & 3.561 \\
\hline $\begin{array}{l}\text { Kinosternidae } \\
\text { Kinosternon herrerai Stejneger, } \\
1925\end{array}$ & tortuga / coxuali & 1.278 & 1.189 & 0.333 & 0.343 & 1 & 4.143 \\
\hline $\begin{array}{l}\text { Anguidae } \\
\text { Gerrhonotus ophiurus Cope, } 1866\end{array}$ & axilis / escorpión & 1.177 & 1.652 & 0.237 & 0.000 & 1 & 4.066 \\
\hline $\begin{array}{l}\text { Corytophanidae } \\
\text { Corytophanes hernandezii } \\
\text { Wiegmann, } 1828\end{array}$ & $\begin{array}{l}\text { lagartija verde / } \\
\text { iguana }\end{array}$ & 1.278 & 1.487 & 0.333 & 0.343 & 1 & 4.441 \\
\hline $\begin{array}{l}\text { Gekkonidae } \\
\text { Hemidactylus frenatus Schlegel, } \\
1836\end{array}$ & lagartija güera / huija & 1.125 & 1.642 & 0.278 & 0.000 & 1 & 4.044 \\
\hline
\end{tabular}

Iguanidae 


\begin{tabular}{|c|c|c|c|c|c|c|c|}
\hline $\begin{array}{l}\text { Ctenosaura acanthura (Shaw, } \\
1802 \text { ) }\end{array}$ & lagartija negra & 0.959 & 0.880 & 0.111 & 0.000 & 1 & 2.950 \\
\hline $\begin{array}{l}\text { Boidae } \\
\text { Boa constrictor (Linnaeus, 1758) }\end{array}$ & $\begin{array}{l}\text { venadillo / } \\
\text { mazatlcoatl }\end{array}$ & 1.177 & 2.065 & 0.296 & 0.914 & 1 & 5.453 \\
\hline \multicolumn{8}{|l|}{ Colubridae } \\
\hline $\begin{array}{l}\text { Coniophanes fissidens (Günther, } \\
\text { 1858) }\end{array}$ & víbora de agua & 1.125 & 1.223 & 0.167 & 0.000 & 1 & 3.515 \\
\hline $\begin{array}{l}\text { Drymarchon melanurus (Duméril, } \\
\text { Bibron y Duméril, 1854) }\end{array}$ & $\begin{array}{l}\text { víbora negra / } \\
\text { tepetlcoatl }\end{array}$ & 1.326 & 2.099 & 0.352 & 0.543 & 1 & 5.320 \\
\hline $\begin{array}{l}\text { Drymobius margaritiferus } \\
\text { (Schlegel, 1837) }\end{array}$ & chirrionera & 0.642 & 0.307 & 0.065 & 0.000 & 1 & 2.011 \\
\hline $\begin{array}{l}\text { Lampropeltis triangulum } \\
\text { (Lacépede, 1788) }\end{array}$ & coralillo / zicatena & 2.169 & 5.298 & 0.607 & 0.195 & 1 & 9.263 \\
\hline $\begin{array}{l}\text { Nerodia rhombifer (Hallowell, } \\
\text { 1852) }\end{array}$ & $\begin{array}{l}\text { víbora de agua / } \\
\text { atlcoatl }\end{array}$ & 1.125 & 1.223 & 0.167 & 0.000 & 1 & 3.515 \\
\hline Spilotes pullatus (Linnaeus, 1758) & apachite / apaxcoatl & 1.830 & 3.297 & 0.574 & 0.886 & 1 & 7.587 \\
\hline Tantilla rubra (Cope, 1876) & coralillo / zicatena & 2.169 & 5.292 & 0.607 & 0.195 & 1 & 9.263 \\
\hline $\begin{array}{l}\text { Tropidodipsas sartorii Cope, } \\
1863\end{array}$ & coralillo / zicatena & 2.169 & 5.292 & 0.607 & 0.195 & 1 & 9.263 \\
\hline \multicolumn{8}{|l|}{ Elapidae } \\
\hline $\begin{array}{l}\text { Micrurus diastema (Duméril, } \\
\text { Bibron y Duméril, 1854) } \\
\text { Viperidae }\end{array}$ & coralillo / zicatena & 2.169 & 5.292 & 0.607 & 0.195 & 1 & 9.263 \\
\hline $\begin{array}{l}\text { Agkistrodon taylori Burger y } \\
\text { Robertson, } 1951\end{array}$ & $\begin{array}{l}\text { cola blanca / } \\
\text { xochicuitlapilii }\end{array}$ & 1.755 & 3.443 & 0.537 & 0.276 & 1 & 7.011 \\
\hline $\begin{array}{l}\text { Atropoides nummifer (Rüppell, } \\
\text { 1845) }\end{array}$ & metlapil & 1.125 & 1.502 & 0.139 & 0.143 & 1 & 3.909 \\
\hline Bothrops asper (German, 1884) & mahuaquite & 1.755 & 3.024 & 0.322 & 0.276 & 1 & 6.377 \\
\hline Crotalus sp. & víbora de cascabel & 1.373 & 1.982 & 0.185 & 0.381 & 1 & 4.922 \\
\hline $\begin{array}{l}\text { BIRDS } \\
\text { Specie }\end{array}$ & Traditional Names & $I_{\text {R-A }}$ & AMO & NT & VTU/PU & $\begin{array}{l}1 \\
\mathbf{O}\end{array}$ & BKSI \\
\hline $\begin{array}{l}\text { Tinamidae } \\
\text { Crypturellus cinnamomeus } \\
\text { (Lesson, 1842) }\end{array}$ & perdiz / xacoyotl & 0.709 & 0.504 & 0.160 & 0.432 & 1 & 2.804 \\
\hline $\begin{array}{l}\text { Anatidae } \\
\text { Cairina moschata (Linnaeus, } \\
\text { 1758) }\end{array}$ & pato negro / azule & 1.023 & 1.661 & 0.262 & 0.000 & 1 & 3.947 \\
\hline $\begin{array}{l}\text { Cracidae } \\
\text { Ortalis vetula Wagler } 1830\end{array}$ & $\begin{array}{l}\text { chachalaca / } \\
\text { ecuetlacte }\end{array}$ & 0.915 & 1.063 & 0.225 & 0.914 & 1 & 4.117 \\
\hline $\begin{array}{l}\text { Penelope purpurascens Wagler } \\
1830\end{array}$ & cojolite / coxolictli & 0.645 & 0.442 & 0.125 & 0.381 & 1 & 2.592 \\
\hline Crax rubra Linnaeus, 1758 & $\begin{array}{l}\text { faisán / pájaro } \\
\text { paisana / pájaro del } \\
\text { cerro / pavo real / } \\
\text { tepetltototl }\end{array}$ & 1.100 & 1.747 & 1.776 & 4.724 & 1 & 10.348 \\
\hline $\begin{array}{l}\text { Odontophoridae } \\
\text { Dendrortyx barbatus Gould, } 1846\end{array}$ & $\begin{array}{l}\text { godorniz / codorniz / } \\
\text { tzoli }\end{array}$ & 0.709 & 0.688 & 0.345 & 0.648 & 1 & 3.390 \\
\hline $\begin{array}{l}\text { Ciconiidae } \\
\text { Mycteria americana Linnaeus, } \\
1758\end{array}$ & cigüeña & 0.434 & 0.269 & 0.0190 & 0.000 & 1 & 1.722 \\
\hline
\end{tabular}




\begin{tabular}{|c|c|c|c|c|c|c|c|}
\hline $\begin{array}{l}\text { Pelecanidae } \\
\text { Pelecanus erythrorhynchos } \\
\text { Gmelin, } 1789\end{array}$ & pelícano & 0.271 & 0.086 & 0.010 & 0.000 & 1 & 1.368 \\
\hline \multicolumn{8}{|l|}{ Ardeidae } \\
\hline Ardea alba Linnaeus, 1758 & garza blanca & 0.315 & 0.121 & 0.016 & 0.000 & 1 & 1.451 \\
\hline Egretta caerulea Linnaeus, 1758 & garza negra & 0.612 & 0.647 & 0.036 & 0.000 & 1 & 2.295 \\
\hline $\begin{array}{l}\text { Cochlearius cochlearius } \\
\text { Linnaeus, } 1766\end{array}$ & cucharita & 0.472 & 0.337 & 0.026 & 0.000 & 1 & 1.835 \\
\hline \multicolumn{8}{|l|}{ Cathartidae } \\
\hline $\begin{array}{l}\text { Coragyps atratus (Bechstein, } \\
\text { 1793) }\end{array}$ & zopilote / tzapilotl & 1.199 & 2.129 & 0.255 & 2.000 & 1 & 6.582 \\
\hline Cathartes aura Linnaeus, 1758 & $\begin{array}{l}\text { zopilote rojo / } \\
\text { zapilotl }\end{array}$ & 0.226 & 0.037 & 0.029 & 0.229 & 1 & 1.521 \\
\hline \multicolumn{8}{|l|}{ Accipitridae } \\
\hline $\begin{array}{l}\text { Buteo magnirostris (Gmelin, } \\
1788 \text { ) }\end{array}$ & pollero & 0.226 & 0.023 & 0.010 & 0.038 & 1 & 1.298 \\
\hline Buteo plagiatus (Schlegel 1862) & $\begin{array}{l}\text { águila / gavilán / } \\
\text { cuatoctle }\end{array}$ & 1.150 & 1.399 & 0.361 & 1.676 & 1 & 5.586 \\
\hline Buteo jamaicensis (Gmelin, 1788) & $\begin{array}{l}\text { águila / gavilán / } \\
\text { cuatoctle }\end{array}$ & 1.150 & 1.399 & 0.361 & 1.676 & 1 & 5.586 \\
\hline \multicolumn{8}{|l|}{ Columbidae } \\
\hline $\begin{array}{l}\text { Patagioenas flavirostris (Wagler, } \\
1831 \text { ) }\end{array}$ & $\begin{array}{l}\text { turcaza / } \\
\text { teczococoteck }\end{array}$ & 0.770 & 0.592 & 0.178 & 0.482 & 1 & 3.023 \\
\hline Zenaida asiática (Linnaeus, 1758) & $\begin{array}{l}\text { paloma blanca / } \\
\text { singuilotl / paloma } \\
\text { de maíz }\end{array}$ & 0.360 & 0.205 & 0.142 & 0.178 & 1 & 1.880 \\
\hline Columbina inca (Lesson, 1847) & tortolita & 0.543 & 0.308 & 0.031 & 0.000 & 1 & 1.882 \\
\hline $\begin{array}{l}\text { Claravis pretiosa Oberholser, } \\
1899\end{array}$ & paloma & 1.269 & 1.788 & 0.026 & 0.965 & 1 & 5.048 \\
\hline $\begin{array}{l}\text { Leptotila verreauxi (Bonaparte, } \\
1855 \text { ) }\end{array}$ & paloma & 1.269 & 1.788 & 0.026 & 0.965 & 1 & 5.048 \\
\hline Zentrygon albifacies Sclater, 1858 & paloma & 1.269 & 1.788 & 0.026 & 0.965 & 1 & 5.048 \\
\hline \multicolumn{8}{|c|}{ Tytonidae } \\
\hline Tyto alba (Scopoli, 1769) & $\begin{array}{l}\text { lechuza / tecolote } \\
\text { lechuza }\end{array}$ & 0.356 & 0.251 & 0.073 & 0.267 & 1 & 1.946 \\
\hline \multicolumn{8}{|l|}{ Strigidae } \\
\hline ? & $\begin{array}{l}\text { cuxcux / tecolote } \\
\text { chico }\end{array}$ & 0.677 & 0.576 & 0.167 & 0.813 & 1 & 3.233 \\
\hline Ciccaba virgata Cassin, 1849 & $\begin{array}{l}\text { tecolote grande / } \\
\text { tecolotl / } \\
\text { cuamocmoctle }\end{array}$ & 1.197 & 1.869 & 0.820 & 1.778 & 1 & 6.669 \\
\hline \multicolumn{8}{|l|}{ Caprimulgidae } \\
\hline $\begin{array}{l}\text { Antrostomus vociferus Wilson, } \\
1812\end{array}$ & $\begin{array}{l}\text { tapacaminos / } \\
\text { poxuaca / } \\
\text { correcaminos }\end{array}$ & 0.508 & 0.231 & 0.241 & 0.0350 & 1 & 2.014 \\
\hline \multicolumn{8}{|l|}{ Trochilidae } \\
\hline & $\begin{array}{l}\text { chuparrosas / colibrí } \\
\text { / huitzil }\end{array}$ & 1.269 & 1.739 & 0.831 & 1.086 & 1 & 5.925 \\
\hline \multicolumn{8}{|l|}{ Momotidae } \\
\hline $\begin{array}{l}\text { Momotus momota (Linnaeus, } \\
1766 \text { ) }\end{array}$ & gorrobano / motmot & 0.271 & 0.098 & 0.047 & 0.000 & 1 & 1.417 \\
\hline \multicolumn{8}{|l|}{ Alcedinidae } \\
\hline $\begin{array}{l}\text { Megaceryle torquata (Linnaeus, } \\
1766 \text { ) }\end{array}$ & $\begin{array}{l}\text { martín pescador } \\
\text { /achachale }\end{array}$ & 0.578 & 0.587 & 0.068 & 0.041 & 1 & 2.274 \\
\hline $\begin{array}{l}\text { Chloroceryle amazona Latham, } \\
1790\end{array}$ & $\begin{array}{l}\text { martín pescador } \\
\text { /achachale }\end{array}$ & 0.578 & 0.587 & 0.068 & 0.041 & 1 & 2.274 \\
\hline
\end{tabular}




\begin{tabular}{|c|c|c|c|c|c|c|c|}
\hline $\begin{array}{l}\text { Ramphastidae } \\
\text { Aulacorhynchus prasinus Gould, } \\
1834\end{array}$ & tucaneta & 0.226 & 0.028 & 0.010 & 0.114 & 1 & 1.379 \\
\hline $\begin{array}{l}\text { Ramphastos sulfuratus Lesson, } \\
1830\end{array}$ & $\begin{array}{l}\text { tucán / pico rial / } \\
\text { tencuahuacalitl }\end{array}$ & 0.271 & 0.098 & 0.102 & 0.317 & 1 & 1.789 \\
\hline \multicolumn{8}{|l|}{ Picidae } \\
\hline$?$ & carpintero & 0.887 & 1.165 & 0.060 & 0.146 & 1 & 3.258 \\
\hline $\begin{array}{l}\text { Melanerpes aurifrons (Wagler, } \\
1829 \text { ) }\end{array}$ & $\begin{array}{l}\text { pájaro carpintero / } \\
\text { cuatzurepetl }\end{array}$ & 0.645 & 0.677 & 0.099 & 0.095 & 1 & 2.516 \\
\hline $\begin{array}{l}\text { Dryocopus lineatus (Linnaeus, } \\
1766 \text { ) }\end{array}$ & $\begin{array}{l}\text { pájaro carpintero / } \\
\text { cuachenche }\end{array}$ & 0.472 & 0.384 & 0.023 & 0.063 & 1 & 1.942 \\
\hline $\begin{array}{l}\text { Campephilus guatemalensis } \\
\text { (Hartlaub, 1844) }\end{array}$ & $\begin{array}{l}\text { pájaro carpintero / } \\
\text { cuachenche }\end{array}$ & 0.472 & 0.384 & 0.023 & 0.063 & 1 & 1.942 \\
\hline \multicolumn{8}{|l|}{ Falconidae } \\
\hline $\begin{array}{l}\text { Micrastur semitorquatus Vieillot, } \\
1817\end{array}$ & vaquero & 0.315 & 0.083 & 0.012 & 0.114 & 1 & 1.524 \\
\hline $\begin{array}{l}\text { Caracara cheriway (Jacquin, } \\
\text { 1784) }\end{array}$ & huatzi & 0.315 & 0.111 & 0.016 & 0.381 & 1 & 1.822 \\
\hline $\begin{array}{l}\text { Herpetotheres cachinnans } \\
\text { (Linnaeus, 1758) }\end{array}$ & guactli & 0.396 & 0.159 & 0.017 & 0.152 & 1 & 1.724 \\
\hline Falco sparverius Linnaeus, 1758 & halcón & 0.271 & 0.051 & 0.013 & 0.095 & 1 & 1.431 \\
\hline \multicolumn{8}{|l|}{ Psittacidae } \\
\hline $\begin{array}{l}\text { Aratinga holochlora (Sclater, } \\
\text { 1859) }\end{array}$ & cotorritos / quilime & 0.770 & 0.918 & 0.178 & 0.482 & 1 & 3.349 \\
\hline Pionus senilis Spix, 1824 & perico / coyo & 0.887 & 1.248 & 0.151 & 0.876 & 1 & 4.163 \\
\hline $\begin{array}{l}\text { Amazona viridigenalis (Cassin, } \\
\text { 1853) }\end{array}$ & perico / cutcho & 0.887 & 1.087 & 0.105 & 0.876 & 1 & 3.956 \\
\hline $\begin{array}{l}\text { Amazona autumnalis (Linnaeus, } \\
\text { 1758) }\end{array}$ & perico / cutcho & 0.887 & 1.087 & 0.105 & 0.876 & 1 & 3.956 \\
\hline \multicolumn{8}{|l|}{ Furnariidae } \\
\hline Xiphorhynchussp. & cuanextototl & 0.356 & 0.128 & 0.018 & 0.000 & 1 & 1.502 \\
\hline \multicolumn{8}{|l|}{ Tyrannidae } \\
\hline $\begin{array}{l}\text { Pyrocephalus rubinus (Boddaert, } \\
1783 \text { ) }\end{array}$ & cardenal & 0.071 & 0.001 & 0.002 & 0.003 & 1 & 1.077 \\
\hline Myiozetetes similis (Spix, 1825) & huiliquitzo & 0.396 & 0.137 & 0.021 & 0.000 & 1 & 1.554 \\
\hline \multicolumn{8}{|l|}{ Corvidae } \\
\hline $\begin{array}{l}\text { Cyanocorax yncas (Boddaert, } \\
1783 \text { ) }\end{array}$ & papán & 0.612 & 0.454 & 0.029 & 0.044 & 1 & 2.139 \\
\hline Corvus imparatus Peters, 1929 & cuervo / cacalotl & 0.578 & 0.347 & 0.122 & 0.660 & 1 & 2.707 \\
\hline \multicolumn{8}{|l|}{ Hirundinidae } \\
\hline Hirundo rustica Linnaeus, 1758 & golondrina & 0.356 & 0.144 & 0.015 & 0.000 & 1 & 1.514 \\
\hline \multicolumn{8}{|l|}{ Turdidae } \\
\hline $\begin{array}{l}\text { Myadestes occidentalis Stejneger, } \\
1882\end{array}$ & jilguero / coyoltototl & 0.127 & 0.029 & 0.019 & 0.006 & 1 & 1.173 \\
\hline Turdus grayi Bonaparte, 1838 & $\begin{array}{l}\text { primavera / } \\
\text { cuitlaltototl }\end{array}$ & 0.830 & 0.792 & 0.197 & 0.067 & 1 & 2.885 \\
\hline \multicolumn{8}{|l|}{ Parulidae } \\
\hline & $\begin{array}{l}\text { chikchik / } \\
\text { zehualtotometl }\end{array}$ & 0.356 & 0.142 & 0.066 & 0.000 & 1 & 1.564 \\
\hline \multicolumn{8}{|l|}{ Icteridae } \\
\hline $\begin{array}{l}\text { Quiscalus mexicanus (Gmelin, } \\
\text { 1788) }\end{array}$ & tordo / acatzana & 1.150 & 1.468 & 0.086 & 1.257 & 1 & 4.962 \\
\hline Molothrus aeneus (Wagler, 1829) & $\begin{array}{l}\text { tordo de ojos rojos / } \\
\text { pixpix }\end{array}$ & 0.578 & 0.548 & 0.122 & 0.495 & 1 & 2.743 \\
\hline Molothrus ater (Boddaert, 1783) & tordo / acatzana & 1.150 & 1.468 & 0.086 & 1.257 & 1 & 4.962 \\
\hline
\end{tabular}




\begin{tabular}{llllllll} 
Icterus sp. & $\begin{array}{l}\text { calandria / pájaro del } \\
\text { sol/tonaltototl } \\
\text { papán real }\end{array}$ & 0.315 & 0.081 & 0.122 & 0.019 & 1 & 1.537 \\
$\begin{array}{l}\text { Psarocolius montezuma (Lesson, } \\
\text { 1830) }\end{array}$ & 0.508 & 0.340 & 0.027 & 0.209 & 1 & 2.086 \\
\hline $\begin{array}{l}\text { Fringillidae } \\
\text { Spinus psaltria (Say, 1823) }\end{array}$ & $\begin{array}{l}\text { dominico / } \\
\text { xochitltototl }\end{array}$ & 0.179 & 0.051 & 0.0281 & 0.000 & 1 & 1.258
\end{tabular}

\begin{tabular}{|c|c|c|c|c|c|c|c|}
\hline $\begin{array}{l}\text { MAMMALS } \\
\text { Specie }\end{array}$ & Traditional Names & MF $_{\text {R-A }}$ & AMO & RNT & VTU/PU & $\mathbf{O}$ & BKSI \\
\hline \multicolumn{8}{|l|}{ Marmosidae } \\
\hline $\begin{array}{l}\text { Marmosa mexicana Merriam, } \\
1897\end{array}$ & $\begin{array}{l}\text { ratón tlacuache / } \\
\text { quimichitlacuaquilotl }\end{array}$ & 0.308 & 0.077 & 0.054 & 0.000 & 1 & 1.438 \\
\hline \multicolumn{8}{|l|}{ Didelphidae } \\
\hline $\begin{array}{l}\text { Didelphis marsupialis } \\
\text { Linnaeus, } 1757\end{array}$ & $\begin{array}{l}\text { tacuache / } \\
\text { tacuaquilotl }\end{array}$ & 1.522 & 3.356 & 0.457 & 1.554 & 1 & 7.889 \\
\hline Didelphis virginiana Kerr, 1792 & $\begin{array}{l}\text { tacuache grade / } \\
\text { itztatacuaquilotl }\end{array}$ & 0.308 & 0.093 & 0.054 & 0.183 & 1 & 1.638 \\
\hline $\begin{array}{l}\text { Philander opossum } \\
\text { (Linnaeus, } 1758)\end{array}$ & zehuantele & 0.368 & 0.141 & 0.015 & 0.000 & 1 & 1.524 \\
\hline \multicolumn{8}{|l|}{ Dasypodidae } \\
\hline $\begin{array}{l}\text { Dasypus novemcinctus } \\
\text { Linnaeus, } 1758\end{array}$ & armadillo / aitoche & 1.461 & 2.568 & 0.430 & 1.829 & 1 & 7.288 \\
\hline \multicolumn{8}{|l|}{ Myrmercophagidae } \\
\hline $\begin{array}{l}\text { Tamandua mexicana (de } \\
\text { Saussure, } 1860)\end{array}$ & $\begin{array}{l}\text { oso mielero / oso } \\
\text { hormiguero / xopa } \\
\text { miel }\end{array}$ & 0.770 & 0.774 & 0.378 & 0.396 & 1 & 3.318 \\
\hline \multicolumn{8}{|l|}{ Orden Insectivora } \\
\hline & ratón / quimichi & 0.532 & 0.2562 & 0.030 & 0.000 & 1 & 1.818 \\
\hline \multicolumn{8}{|l|}{ Orden Chiroptera } \\
\hline \multicolumn{8}{|l|}{ Canidae } \\
\hline Canis latrans Say, 1823 & $\begin{array}{l}\text { coyote / perro coyote } \\
\text { / coyotl / coyochichi }\end{array}$ & 1.492 & 2.921 & 1.675 & 2.829 & 1 & 9.916 \\
\hline $\begin{array}{l}\text { Urocyon cinereoargenteus } \\
\text { (Schreber, 1775) }\end{array}$ & $\begin{array}{l}\text { zorro / cayochi / } \\
\text { itzcayuchi }\end{array}$ & 1.129 & 1.964 & 0.640 & 0.880 & 1 & 5.613 \\
\hline \multicolumn{8}{|l|}{ Felidae } \\
\hline $\begin{array}{l}\text { Herpailurus yagouaroundi } \\
\text { (Lecépède, 1809) }\end{array}$ & $\begin{array}{l}\text { onza / sacamixtle / } \\
\text { yaguarundi }\end{array}$ & 0.425 & 0.210 & 0.175 & 0.067 & 1 & 1.879 \\
\hline $\begin{array}{l}\text { Leopardus pardalis (Linnaeus, } \\
1758 \text { ) }\end{array}$ & ocelote / leoncillo & 0.425 & 0.224 & 0.081 & 0.091 & 1 & 1.821 \\
\hline Leopardus wiedii (Schinz, 1821) & $\begin{array}{l}\text { tigrillo / } \\
\text { cuametamixto }\end{array}$ & 1.165 & 2.391 & 0.217 & 1.533 & 1 & 6.306 \\
\hline Lynx rufus (Schreber, 1777) & $\begin{array}{l}\text { gato montés / gatillo } \\
\text { / cuametamixto }\end{array}$ & 0.725 & 0.683 & 0.277 & 0.549 & 1 & 3.234 \\
\hline Puma concolor (Linnaeus, 1771) & lión / tecuani & 0.632 & 0.539 & 0.037 & 0.076 & 1 & 2.284 \\
\hline Panthera onca (Linnaeus, 1758) & tigre / tecuani & 0.898 & 1.181 & 0.119 & 0.122 & 1 & 3.320 \\
\hline \multicolumn{8}{|l|}{ Mustelidae } \\
\hline Eira barbara (Linnaeus, 1758) & $\begin{array}{l}\text { perro de cerro / } \\
\text { tepechichi }\end{array}$ & 0.632 & 0.426 & 0.134 & 0.076 & 1 & 2.268 \\
\hline \multirow{2}{*}{$\begin{array}{l}\text { Galictis vittata (Schreber, 1776) } \\
\text { Mustela frenata Lichtenstein, } \\
1831\end{array}$} & gato grisón & 0.098 & 0.007 & 0.003 & 0.004 & 1 & 1.112 \\
\hline & hurón & 0.368 & 0.143 & 0.015 & 0.057 & 1 & 1.583 \\
\hline
\end{tabular}

Mephitidae 


\begin{tabular}{|c|c|c|c|c|c|c|c|}
\hline $\begin{array}{l}\text { Conepatus leuconotus } \\
\text { (Lichtenstein, 1832) }\end{array}$ & zorrillo / epa & 1.551 & 3.230 & 0.190 & 1.600 & 1 & 7.572 \\
\hline $\begin{array}{l}\text { Mephistis macroura Lichtenstein, } \\
1832\end{array}$ & zorrillo / epa & 0.425 & 0.185 & 0.033 & 0.274 & 1 & 1.917 \\
\hline Spilogate gracilis Merriam, 1890 & zorrillo / epa & 0.425 & 0.185 & 0.033 & 0.274 & 1 & 1.917 \\
\hline \multirow{4}{*}{$\begin{array}{l}\text { Procyonidae } \\
\text { Potos flavus (Schreber, 1774) } \\
\text { Nasua narica (Linnaeus, 1776) } \\
\text { Procyon lotor (Linnaeus, 1758) }\end{array}$} & \multirow{4}{*}{$\begin{array}{l}\text { marta / tancho } \\
\text { tejón / pezoctle } \\
\text { mapache / mapachi / } \\
\text { cuametamixto }\end{array}$} & & & & & & \\
\hline & & 0.632 & 0.482 & 0.134 & 0.229 & 1 & 2.477 \\
\hline & & 1.824 & 2.950 & 0.604 & 1.371 & 1 & 7.750 \\
\hline & & 1.609 & 3.276 & 0.855 & 1.128 & 1 & 7.867 \\
\hline \multirow{3}{*}{$\begin{array}{l}\text { Cervidae } \\
\text { Mazama americana (Erxleben, } \\
\text { 1777) } \\
\text { Odocoileus virginianus } \\
\text { (Zimmermann, 1780) } \\
\end{array}$} & \multirow{3}{*}{$\begin{array}{l}\text { venado chico / } \\
\text { cuachacal / chacal } \\
\text { venado / mazatl }\end{array}$} & & & & & & \\
\hline & & 0.856 & 0.825 & 0.470 & 1.200 & 1 & 4.351 \\
\hline & & 1.849 & 3.604 & 0.687 & 3.680 & 1 & 10.82 \\
\hline \multicolumn{2}{|l|}{ Tayassuidae } & & & & & & \\
\hline Tayassu tajacu (Linnaeus, 1758) & jabalí / coapitzotl & 1.8991 & 3.526 & 0.716 & 2.194 & 1 & 9.336 \\
\hline $\begin{array}{l}\text { Sciuridae } \\
\text { Sciurus aureogaster Cuvier, } 1829\end{array}$ & ardilla / tecomate & 1.665 & 2.938 & 0.524 & 1.189 & 1 & 7.316 \\
\hline $\begin{array}{l}\text { Geomyidae } \\
\text { Orthogeomys hispidus (Le Conte, } \\
\text { 1852) }\end{array}$ & $\begin{array}{l}\text { tuza / tuza de tierra / } \\
\text { tuza raicera / } \\
\text { tlatltuza }\end{array}$ & 1.367 & 2.786 & 1.472 & 0.055 & 1 & 6.680 \\
\hline Muridae & ratón / quimichi & 0.532 & 0.256 & 0.060 & 0.000 & 1 & 1.848 \\
\hline $\begin{array}{l}\text { Erethizontidae } \\
\text { Coendu mexicanus (Kerr, 1792) }\end{array}$ & puerco espín / xompi & 1.235 & 2.213 & 0.336 & 0.381 & 1 & 5.165 \\
\hline $\begin{array}{l}\text { Cuniculidae } \\
\text { Cuniculus paca (Linnaeus, 1776) }\end{array}$ & tuza real / cuatuza & 1.200 & 1.890 & 0.358 & 0.549 & 1 & 4.996 \\
\hline $\begin{array}{l}\text { Leporidae } \\
\text { Sylvilagus brasiliensis (Linnaeus, } \\
\text { 1758) }\end{array}$ & conejo / cuatochi & 1.824 & 4.323 & 0.604 & 1.029 & 1 & 8.780 \\
\hline
\end{tabular}

\subsection{Mention frequency Rank Abundance}

The species that receive the highest values in this sub-index are: a) mammals Pecari tajacu (collared peccary) with a value of $\mathrm{MF}_{\mathrm{R}-\mathrm{A}}=1.8$ with 48 mentions; $\mathrm{b}$ ) in birds the complex of species known by the generic name of pigeons (Claravis pretiosa, Leptotila verreauxi, Geotrygon albifacies; $\mathrm{MF}_{\mathrm{R}-\mathrm{A}}=1.2, \mathrm{n}=38$ ), as well as the Trochilidae family ('huitzil/hummingbird', with the same values); c) for reptiles the 'coral' complex (Lampropeltis triangulum, Tantilla rubra, Tropidodipsas sartorii and Micrurus diastema; $\left.M_{R-A}=2.1, n=41\right)$; ) in a group of river fish or animals, the highest value is for the 'cosol' ethnospecies corresponding to a river crustacean, for which its taxonomic identification was not possible $\left(\mathrm{MF}_{\mathrm{R}-\mathrm{A}}=1.8, \mathrm{n}=47\right)$; e) Fungi with the ethnospecies 'xochilnanacatl' integrated by the species Cantharellus cibarius, C. lateritius, $C$. odoratus; $\mathrm{MF}_{\mathrm{R}-\mathrm{A}}=2.8, \mathrm{n}=26$ ); and $\mathrm{f}$ ) for plants, the 'cedar' (Cedrela odorata; $\mathrm{MF}_{\mathrm{R}-\mathrm{A}}=1.3, \mathrm{n}=42$ ). 


\subsection{Average Mention Order}

For this subscript, the species with the highest values are: Odocoileus virginianus $(\mathrm{AMO}=$ 4.3; mammals), Cairina moschata $(\mathrm{AMO}=2.1$; birds $)$ and Cedrela odorata $(\mathrm{AMO}=1.1$;

plants); and for the ethnospecies: 'coralillo' $(\mathrm{AMO}=5.2$, reptiles $)$, 'cosol' $(\mathrm{AMO}=5.29$, crustaceans) and 'xochilnanacatl' ( $\mathrm{AMO}=8.7$, fungi). The similarity between the species in this subscript and in the previous one, is a function of the partial and significant correlation $(\mathrm{r}=0.6040, \mathrm{p}>0.05)$ between both metrics (FMR-A and AMO).

\subsection{Richness Names Traditional}

To calculate this subscript, the nomenclatural association types were first identified, where we have that $50 \%$ of the recognized species in the sample have an equitable association, that is, the corresponding of a species to its ethnospecies perfectly identified (1:1), 37\% have a proportional relationship, more than one traditional name associated with a species $(>1: 1)$, which are generally two names, one in Spanish and the other in Náhuatl; $10 \%$ have more than one traditional name related to more than one species $(>1:>1$; synonymy) and $3 \%$ have a relation of one name to more than one species (1:>1; homonymy). On the other hand, the values of linguistic interpretation (the value that each name receives depending on its meaning) correspond to 118 species named under morphological criteria, 42 species for cultural aspects, 25 species for behavioral characteristics, 19 species for ecological aspects and 30 species are a mixture of some of the previous ones.

The species that presented the highest wealth value of traditional names of each biological group are mammals: Canis latrans with four traditional names (nt) 'coyote/perro coyote/coyotl/coyochichi' $(\mathrm{RTN}=1.6)$; birds: Crax rubra, nt $=5$, 'faisan/pájaro paisana/tepetltototl/pájaro del cerro/pavo real' $(\mathrm{RTN}=1.7)$; reptiles the complex Lampropeltis triangulum, Tantilla rubra, Tropidodipsas sartorii and Micrurus diastema, nt $=2$, 'coralillo/tzicatena' (RTN=0.6); fish: the complex Ictalurus mexicanus, I. puntatus and Pylodictis olivaris, $\mathrm{nt}=4$, 'bagre/xolotl/miquecuani/xolomichi' (RTN= 2.1); fungi: the complex Auricularia auricula, A. delicata, A. fuscosuccinea, nt $=5$, 'oreita/orejita de abuelita/cuapetachiquinte/cualeleshtli/chocoyos'; and for plants: Delonix regia, $\mathrm{nt}=5$, 'framboyán /flor de mayo/flor de las madres/framboyancuahuitl/framboyanxuchitl' (RTN= $0.20)$. 


\subsection{Value Total Uses and Parts Used}

The $90 \%$ of total flora, fauna and microbiota species are within any use category, sometimes, shared by different biological groups, for example, the categories for fauna are: ceremonial, food, pet, medicinal, ornamental and for commerce; some of such are as well shared by fungi and plants; for plant group, the added categories are: fuel, building materials, agricultural use, crafting, fodder, wood and toxic. The used parts of animals are: head, bones, skull, blood, fat, fur, hair, feathers, antlers, carapaces, feet, tail, teeth, bird peaks, eggs, rattles and whole individuals; meanwhile those for plants area: root/bulbs, stem, leaves, flower, fruits, bark, latex/sap, branches and trunks.

Sub index values are a function of the biological group, since each of them displays a determined number of categories and used parts. For example, for mammals, there are 7 use categories and 15 used parts, for birds 7/9, reptiles 7/6, fish 5/2, fungi $2 / 1$ and plants 14/12. The highest values of this sub index belong to Odocoileus virginianus (mammals: VTU/PU= 3.6), Crax rubra (birds: VTU/PU= 4.4), Boa constrictor (reptiles: VTU/PU=0.9), the complex of Ictalurus mexicanus, I. puntatus y Pylodictis olivaris (fish: VTU/PU= 2.6), Cantharellus cibarius, C. lateritius, C. odoratus (fungi: VTU/PU=5.2) and Pimenta dioica (plants: VTU/PU=0.9).

\subsection{Species Origen}

In order to obtain this sub index, the sum of sub index was obtained and added a value of 1 for native species/ethnospecies, while this value of 1 was subtracted for those species identified as introduced or naturalized. The identification contained 50 introduced species, of which five correspond to fish (Amatalania nigrofasciata; 'mojarra', Oreochromis aureus, O. niloticus 'tilapia', Cyprinus carpio communis 'carpa' and Ictalurus puntatus 'bagre') and 45 plant species, of which $25 \%$ are fruit species of economic importance (e.g. mango, tamarind, peach, lime, grapefruit, lychee, pineapple, watermelon, banana, plantain, starfruit, maroon orange, grape); $25 \%$ are edible species but not fruits, $9 \%$ of species are monocultures: coffee (Coffea arabica), sugar cane (Saccharum officinarum), orange (Citrus $\times$ sinensis), lemon (Citrus limon) and tangerine (Citrus reticulata); whilst the remaining $43 \%$ is comprised by different species of trees, herbs and flowers. 


\subsection{Relationship between sub-indexes}

In order to determine the redundancy between the sub-indexes that integrate the BKSI, a multiple lineal correlation between the final sub index value and the sub-indexes, resulting in significant values for $\mathrm{MF}_{\mathrm{R}-\mathrm{A}}(\mathrm{r}=0.93, \mathrm{p}<0.05)$ and $\mathrm{AMO}(\mathrm{r}=0.93, \mathrm{p}<0.05)$. Sub-indexes RNT and VTU/PU are found to have a moderate correlation (RNT: $r=0.79, p<0.05$; VTU/PU: $\mathrm{r}=0.86, \mathrm{p}<0.05)$, being the less correlated sub-index, the origin $(\mathrm{r}=0.38, \mathrm{p}=0.05)$. Redundancy between sub-indexes is a function of the nature of data, for example, the case of MFR-A and AMO, dependence is based in the constant: mentions of $i$ species; while between RNT and VTU/PU this is based on Amorozo \& Gély (1988) correction factor, that is why the last sub-index totally dependent is the species origin. However, these redundancies, not necessarily mean that similar aspects are being evaluated; since there is a constant as part of the metrics between the sub-indexes, there are also other different elements that integrate them; that is why each of the sub-indexes contribute to the index (BKSI) with a different value. Such phenomenon is displayed in the cluster analysis, in which the aim was to represent the relationship between the sub-indexes, displaying a different response than the multiple lineal correlation, being the less correlated AMO and $\mathrm{O}$, meanwhile there is a greater link between RNT and VTU/PU, as well as MFR-A, which indicates that such sub-indexes are independent metrics (Figure 2).

\subsection{Biocultural Key Species Index}

Results obtained from the Biocultural Key Species Index (BKSI) varied from -0.03 to 19.07; the highest value corresponded to a fungi species complex integrated by Cantharellus cibarius, C. lateritius y $C$. odoratus; which constitute the 'hongo amarillo/xochilnanacat1/cuapetachiquinte' ethnospecies; while the lowest values accounted for 48 plant species. Since there is a wide range between the highest and lowest values and by including various biological groups, as well a few sub-indexes comprising the BKSI, it might result in subjective outcomes. Therefore, a selection of the biocultural species (BS) was established, rather by means of a biocultural relevance degree classification than by the obtained value directly. 


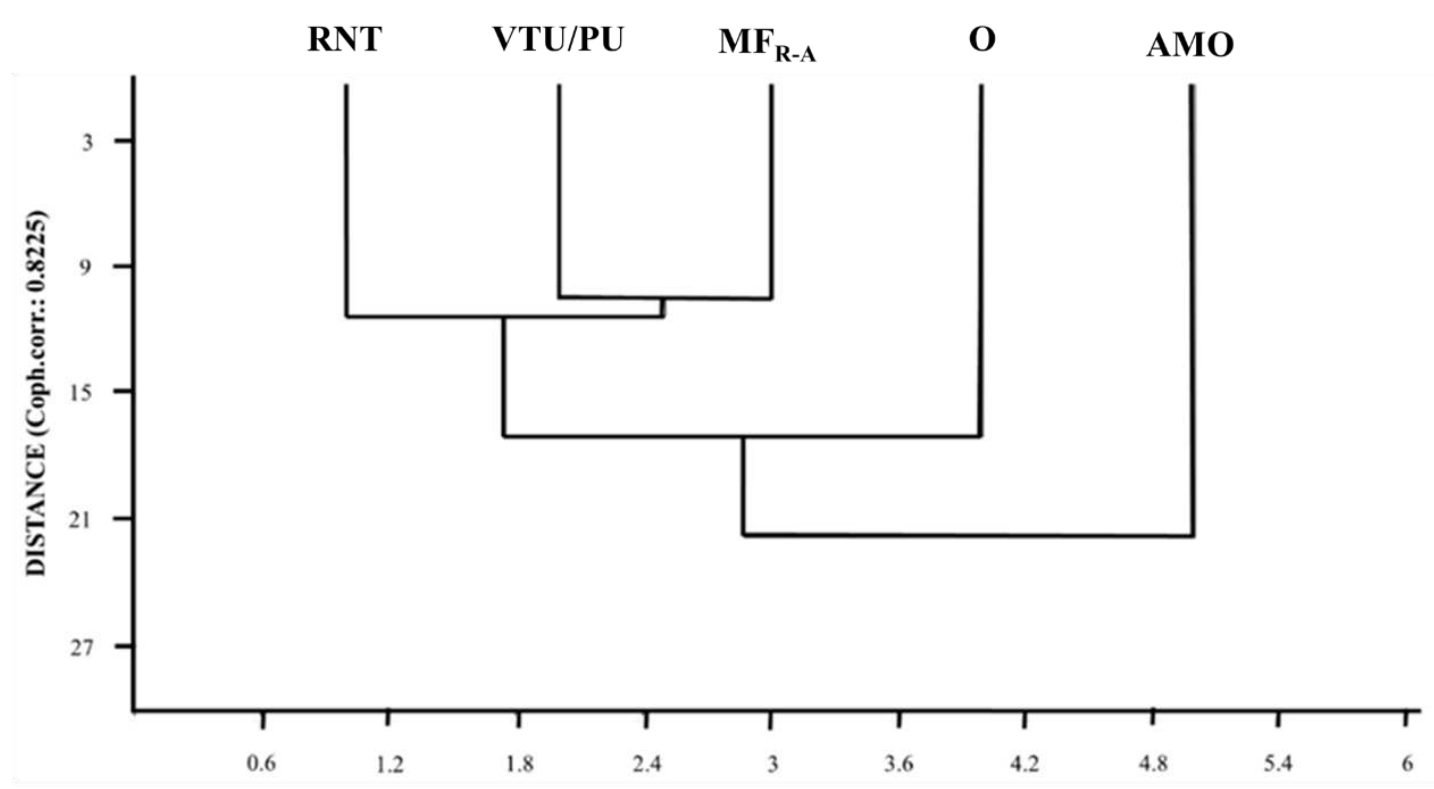

Figure 2. Cluster analysis by means of Euclidean distance grouping. RNT: Richness Names Traditional; VTU/PU: Value Total Uses/Parts Used; MFR-A: Mention Frequency by RankAbundance; O: Species Origin; AMO: Average Mention Order.

Different degrees of biocultural relevance allow for visualization and selection of BS as a function of the obtained value in the index, of the data origin, biocultural relevance, as well as the distribution within the sample (Figure 4). There are four categories of biocultural relevance: Maximum Biocultural Relevance (MaxBR) with a 4\% $(n=15)$; High Biocultural Relevance (HBR; 5\%, $n=17)$; Medium Biocultural Relevance (MBR) with a 53\% (n=197), being the most represented level, and Low Biocultural Relevance (LBR) with a 38\% $(n=141)$.

For the mammal's group, two species were recorded for the MaxBR, which were Odocoileus virginianus ('venado', BKSI=10.8) and Canis latrans ('coyote', BKSI=9.9); in the HBR category there were 4 species: Pecari tajacu ('jabali', BKSI=9.3), Sylvilagus brasiliensis ('conejo', BKSI=8.7), Didelphis marsupialis ('tlacuache', BKSI= 7.8) y Procyon lotor ('mapache', BKSI= 7.8). Those species reported in the first level account for the highest frequency mention values, as well as the most traditional names, use categories and used parts. In the MBR, there are 9 species and in LBR 19 species; in these two categories there 
are medium and small sized species, which are infrequent and do not have a relevant role, according to their use. Overall, all mammal species are important as food source, except for Eira barbara ('tepechichi/viejo de monte'), in tradicional medicine, its use as a pet and by being quoted as ornaments (fur, bones, dissected specimens; Figure 3a).

For the birds, there was only one species within the MaxBR category: Crax rubra ('faisán', $\mathrm{BKSI}=10.3)$, this species is relevant because of its role in traditional medicine, it is quoted by its beauty, it is the most valued bird in terms of its use as food source, ornamental and as pet; however, is a rare species in the area and it is classified as threatened (NOM-059SEMARNAT 2010). In regards of the distribution of data in this biological group, there was none species/ethnospcies within the HBR category. For the MBR values regarding 10 species (Ciccaba virgata, $\mathrm{BKSI}=6.6$; Coragyps atratus $\mathrm{BKSI}=6.5$; Trochilidae, $\mathrm{BKSI}=5.9$; Buteo plagiatus and B. jamaicensis $\mathrm{BKSI}=5.5$; Claravis pretiosa, Leptotila verreauxi and Geotrygon albifacies; BKSI= 5; Quiscalus mexicanus and Molothrus ater; BKSI=4.9); in this category, such species are important because of their traditional use in medicine; for example, healing properties against rabies are attributed to vultures (Coragyps atratus), thus their meat is consumed in broth, besides they are used in magical-religious practices. There are other important species in terms of perception, for example, the owl (Ciccaba virgata/'tecolote'), which represents duality and is attributed with unnumbered magical and healing properties, hence, many of its parts are used. Other examples are Buteo jamaicensis y B. plagiatus, ('águila/cuautli'), these, as well as other predaceous species are commerced as pets and ornamental ones. Nevertheless, unlike other predaceous species documented, these are also associated with exceptinoal magical proterties, in which if a person performs a 'cleaning' with these animals, they (users) can obtain the maximum protection possible, that is why many of their parts are used, as well as whole specimens. Finally, the LBR category is the most representative, with 50 species (Figure $3 \mathrm{~b}$ ).

The herpetofauna group is represented in the MaxBR level by one snake species named 'coralillo' or 'tzicatena', which is considered among the most venomous, its name refers to it as the 'mother of all venomous snakes'. Such ethnospecies comprises four snake species (Lampropeltis triangulum, Tantilla rubra, Tropidodipsas sartorii, Micrurus diastema; BKSI=9.2), of which only one is venomous (Micrurus diastema), unlike the other snakes (viperids) recorded as well, it possesses one of the most potent venoms, which is neurotoxic 
and directly affects the central nervous system, therefore, several species that display morphological similarities are known by the same name and the same cultural connotation is assigned to them. Within the HBR level there are two species: Spilotes pullatus ('apachite', BKSI = 7.5) and Agkistrodon taylori ('cola blanca/xochilcuitlapilli', BKSI=7), such species have a high $\mathrm{MF}_{\mathrm{R}-\mathrm{A}}$ and AMO, in the case of Agkistrodon taylori, which is a highly venomous snake used in traditional medicine, there is great fear around it because of the consequences of being biten by one, since its miotoxical venom causes large damage on the tissues. Accounting for these same features Bothrops asper ('mahuaquite', BKSI=6.3) can be listed, in which traditional nomenclature for 'mahuquite' is referred to a biological characteristic of this species, which means 'deadly progressive infection', however, this is pecies is now in the MBR category along with 3 other snake species. Within the LBR category, there are lizards, turtles and amphibians grouped (10 species). What can be asured is that snakes are classified within the highest categories, by means of being considered harmful to the people (Figure 3c).

Fish constitute an important resource for the community, generally these are species important as a food source and directly sold, ethnospecies 'bagre'and 'cosoles' ('bagre': Ictalurus mexicanus, Pylodictis olivaris; 'cosoles'; BKSI=12.8) are within the MaxBR category. Meanwhile in the HBR category, there are 5 species, from which 'techichis' (Procambarus sp., BKSI=10.4) outstands, as well as 'cosoles', which are both crawfish. There are 11 species in the MBR cateogry and 6 for the LBR (Figure 3d).

Fungi are the less diverse group, however, the 'hongo amarillo/xochilnanacatl' complex $(B K S I=19)$ reached its maximum value (MaxBR). For the HBR category it was not possible to obtain data, while in the MBR the 'hongo blanco' complex (Pleurotus albidus, P. djamour; BKSI= 9.4) and the 'orejitas' (Auricularia auricula, A. delicata, A. fuscosuccinea; BKSI= 9.4) were categorized, as well as two species in the LBR category (Schizophyllum commune, $\mathrm{BKSI}=2.1$; Ustilago maydis, $\mathrm{BKSI}=2$; Figure 3e).

The plant group was the most diverse, with only two species in MaxBR (Psidium guajava, $\mathrm{BKSI}=3.8$; Cedrela odorata, $\mathrm{BKSI}=3.6$ ). For HBR, six species (Pimenta dioica, Persea americana, $\mathrm{BKSI}=3.1 ;$ Pouteria sapota, $\mathrm{BKSI}=3 ;$ Erythrina americana, Bursera simaruba, BKSI= 2.9; Spondias purpurea, BKSI=2.3); these particular species are native trees. The $72 \%(\mathrm{n}=158)$ fall into the MBR category, which is the most diverse and it is characterized 
by including herbs, shurbs and trees with importance as a food source, medicinal, ornamental, fuel source, ceremonial and commercial; some of thesespecies are very important at a cultural level, for exmample, Tagetes erecta ('cempoal', BKSI=2.3), which is herbaceous and its inflorescences and flowers use is very important in the 'xantolo' (dead's day) festivities, besides to perform certain rituals in other times of the year. Two species with a high importance as wood source are Dalbergia palo-escrito ('palo escrito', $\mathrm{BKSI}=1.9$ ) and Ulmus mexicana ('palo varón', BKSI=1.3). The remaining 24\% is grouped within the LBR cateogry. The sample distribution displays certain particularity, overall the values for the plant group are lower when applying the index, since this is a group with a high species richness and the resulting index value is less when incrementing the sample number (Figure 3f).

\section{Discussion}

The present study was focused on evaluating the biocultural relevance of known and used fauna, flora and microbiota species from a Náhuatl community at the Hidalgo Huastec region, Mexico (Figure 4). In general, the studies focused on evaluating cultural importance have dealt with specific biological groups with own features, e.g. edible plants [12], medical important plants [62], economically important plants [14] or edible fungi [13]; which has allowed to recognize cultural importance in a particular way. In this study, by including several biological groups (plants and fungi; mammals, birds, reptiles, amphibians and fish) it was possible to interpret in an overall manner the biocultural relevance as a function of known and used biodiversity. It has been observed that the variables integrating the BKSI act in an impartial way on the biological groups, that is, these confer the same weight or consideration to either one or other group, independently of the species numbers for each; variation in the index values depend on the granted features for each species (e.g. frequency of mentions per biological group, as well as count of traditional names, uses and parts used; and there is not any biological group more relevant than the others, therefore the structure of natural ecosystems for this indigenous group operate as a whole. 

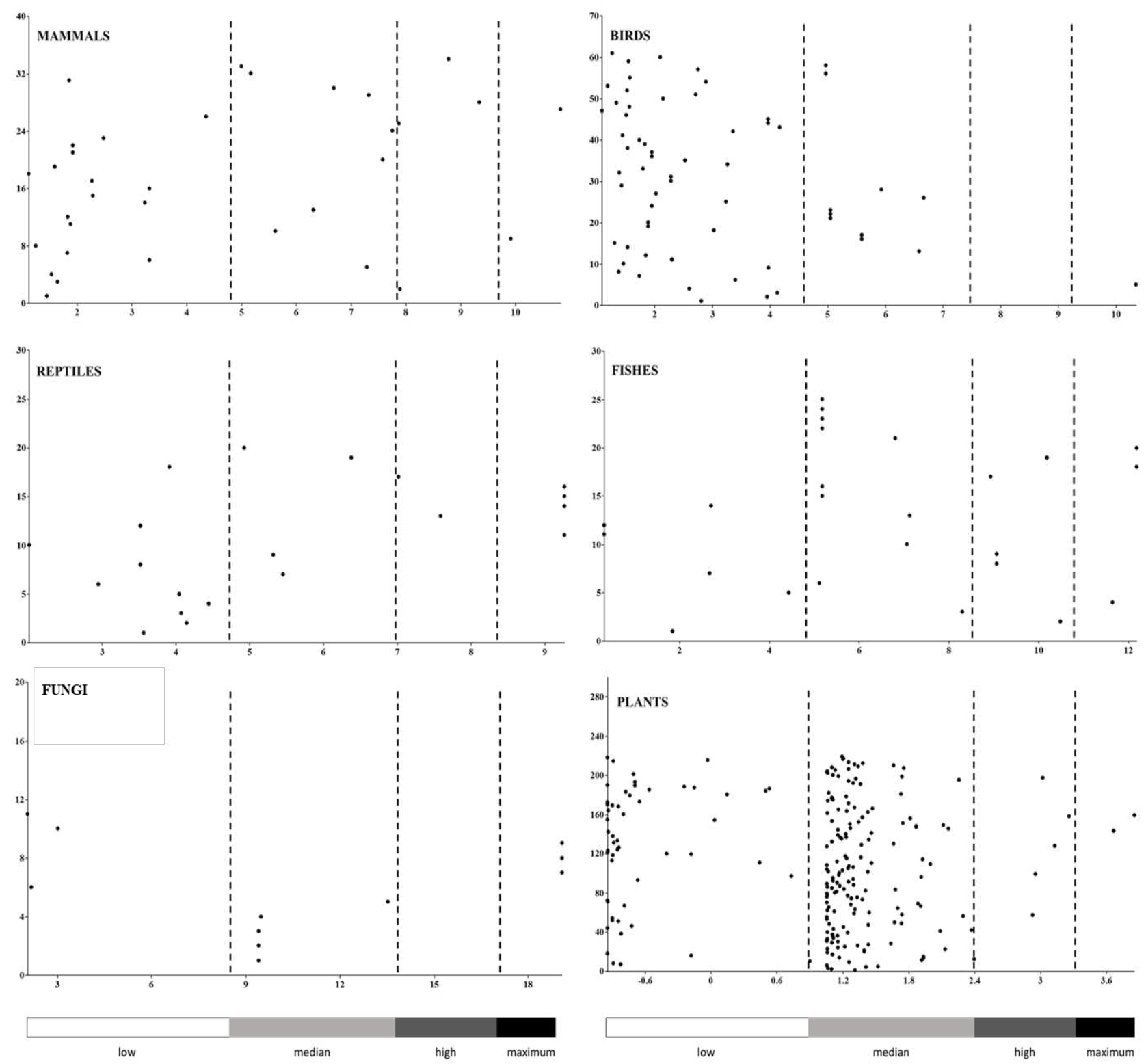

Figure 3. Data dispersion for the BKSI in the six species groups: a) mammals, b) birds, c) reptiles, d) fishes, e) fungi and f) plants; associated in different biocultural relevance level, which are four: MaxBR: Maximum Biocultural Relevance, HBR: High Biocultural Relevance, MBR: Medium Biocultural Relevance, LBR: Low Biocultural Relevance.

It has been found that some biological groups are comprised of a high richness but some do not, this depends directly on the biological characeristics of each group, however, even though some are more diverse than others, it does not necessarily means that they have a higher biocultural relevance, for example, although plants display a higher species with 
respect to fungi, the latter are not less relevant than plants, both groups are evenly known and employed; as usual, ethnobotany studies report a high species that are known and used in distinct ways [3], which follows the same pattern as this present work. Regarding fungi there are studies in different zones of the country that report a considerable number of known and employe species [63-65], in this work there is a report of three species complexes (Cantharellus spp., Pleurotus spp., Auricularia spp.), possibly because many fungi species do not have any use, but the few species are known by most of the inhabitants and the types of use are uniform; besides these are the species recognized and utilized at tropical regions [66]. There are other phenomenon that indicate that species richness is not related to biocultural relevance, e.g. for mammals there is a report of approximately 150 species for this zone [67], in this study the report is of only 31 species, which has to do with species that are not identified at detail culturally, as it is the case for bats (Chiroptera) and mice (Rodentia), where many species simply integrate one ethnospecies; another example is seen for birds, in which species from the Torquilidae are assigned with the same traditional name ('colibrí/huitzltil').

Cultural importance must be evaluated from different parameters that include cultural domain diversity; since the metrics used in enthnobiology, only encloses a fraction of what it belongs to something more inclusive. It must be considered that species can become more or less relevant through the adaptive processes or their participation within the distinct cultural domains or anthropocentric categories, which involve a whole series of social or cultural practices [19]. Therefore, species that obtain the maximum and high relevance values, must be those that have an important role in religion, myths, arts, rituals, politics, science, economy, customs, language, etc. [17-20]. The index used here is merely integrated by five sub-inexes that cover a small fraction of all cultural domains but provide a close look to the cognitive, perceptual and employment of biotic resources aspects. 


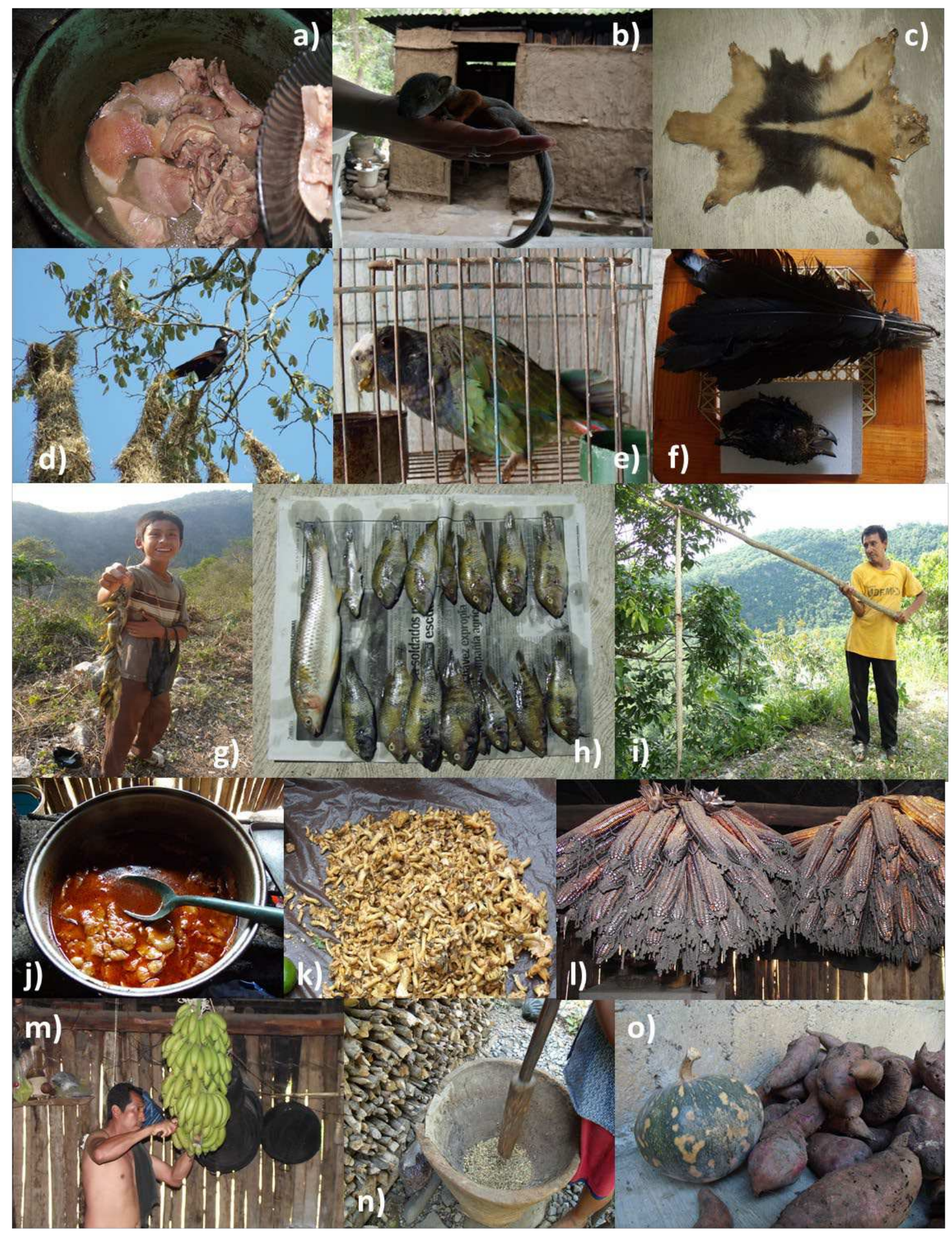

Figure 4. a) royal gopher meat ('tuza real', Cuniculus paca), b) baby squirrel (Sciurus deppei), c) anteater skin ('xopamiel/brazo fuerte' Tamandua mexicana), d) royal papán 
('papán real', Psarocolius montezuma), e) parakeet ('cutcho', Pionus senilis), f) head and feathers of Crax rubra ('pájaro paisana/tepetlototl'), g) fisherman child, h) fish ('poxta', Cichasoma labridens, Herichthys tamasopoensis, Agonostomus montícola), i) Botrops asper ('mahaquite'), j) Pleurotus albidus ('itztacnanacatl/hongo blanco' traditional food), k) 'xochilnanacatl/hongo amarillo' (Cantharellus spp., yellow mushroom), 1) corn cobs (Zea mays), m) banana stalk (Musa paradisiaca), n) firewood and coffee (Coffea arabica), o) cucurbit and sweet potato (Cucurbita máxima and Ipomoea batatas).

Each of the sub-indexes indicate the relationship between species/ethnospecies within an anthropocentric category. For example, sub-indexes MFR-A and AMO indicate the status of $i$ species at a cultural level [9], as well as the knowledge people have of themselves [16]; RNT is a variable of the cultural domain of the language, besides is a primary cognitive construction form, it is worth mentioning that this is the first parameter that attemps to measure this cultural aspect, integrating several nomenclatural relations, following and complementing the bases proposed by Freire \& Pauly [58]; and the VTU/PU includes in a general manner several cultural domains that can be: food source, medicinal, economical, religious, artistic and ritual; therefore, this sub-index provides a practical cultural value [14]. Meanwhile the species origin (O) is an assignment to subtract relevance to introduced species that frequently are important economically, which does not necessarily mean that these are important culturally.

The sub-indexes displayed a different behavior, as like in previous works [9-17]. However, there was a positive correlation, being a constant the number of times a species $i$ was mentioned within the sample (FC; [56]), which in turn is essential because it avoids the overestimation of values. On the other hand, the sub-indexes RNT and VTU/PU display a multiplicity character, since when the number of traditional names for a species $i$ increases, the number of categories and or its employed parts attain higher values. Those species reported with the highest values for each sub-index are overall the same within each biological group, but the order depends on the sub-index type. It is also observed that when the number of species for each biological group is smaller, the larger is the value they obtain for the BKSI and vice versa; thus, there is a relation between the sample number and the 
obtained values, although this is not an indicative of a higher or lower biocultural relevance, instead it is simply a proportional relationship to the sample number.

\subsection{Discussing the sub-indexes}

Sub-indexes MFR-A and AMO are correlated to each other, they both depend on the number of times the informants mention species $i$, so both indexes indicate the degree of knowledge of $i$ species [9, 12, 16], being a good indicator of its cultural value, showing higher homogeneity when a species $i$ is named, as a reflection of being more known by people and therefore being more important [9]. Another aspect indicating that MFR-A is the ecological importance of species $i$, it is supposed that by being the most named, this is more abundant in an ecosystem [9], however, it is considered that this is not necessarily this way, since to sustain such statement it is necessary to perform ecological-ethnobiological studies, besides ethnobiological ones, which will allow to know the ecological status of species $i$. Turner [9] mentions that in an ethnobiological study there are species widely mentioned and others not so much, that can be a reflection of its ecological status; however that is about a relationship between species that can be more mentioned than others; this can possibly or not reflect such ecological role of a species $i$, what is truly appropriate to consider is that such relation between abundant species and rare (as it is done in ecology), thus, an adjustment has been made to the $\mathrm{MF}_{\mathrm{R}-\mathrm{A}}$ sub-index by employing the abundance range curve formula [57], obtaining the proportion of most abundant and rare species in this way, consequently frequency of mentions per abundance range ( $\left.\mathrm{MF}_{\mathrm{R}-\mathrm{A}}\right)$.

In the AMO sub-index, there has been a methodological problem identifie, which has to do directly with the data origins; the premise of this sub-index is that the mentions of the ethnotaxa or species in a list area randomized, and the higher the sample (informant number) and the number of times species $i$ is mentioned, a higher average number will result for species $i$; technically, if two ethnotaxa or species are mentioned the same amount of times but in distinct positions, the predominant of one of the two, is that which has been mentioned in the last spots, due to a growing value assignment. This sub-index was not able to be corrected, despite trying diverse adjustments, because of this, it was decided that by doing a minor adjustment, that allows for lowering the final value and as a result to reduce the overestimation of species $i$. 
Based on the basic principles of traditional classification [1,2] a sub-index that allows for measuring richness of names was designed, implementing a series of concepts, which makes this sub-index subjective $[9,12,13]$. This subscript assesses the role that biotic resources play within language $[17,19,20]$. Being the first subscript of this nature, and provides a quantitative value (by the number of traditional names assigned to species $i$ ) and subjective $[9,12,13]$ when the values are weighted. which corresponds to the linguistic interpretation of each traditional name.

The cultural importance has been strongly operated from the methodological proposal of use value $[10,11,14$ 16], where Reyes-García et al. [14] indicates that his is a way to measure active knowledge of a species $i$. The sub-index (VTU/PU) integrates both the uses given for a species $i$, as well as the employed parts of such species.

\subsection{Species with high biocultural relevance}

Overall, one of the most significant variables to determine the biocultural relevance is the employment given to species, e.g. for fungi there were two use categories, while there were twelve for plants; it is worth mentioning that cultural importance has been originated through the measuring of the associated uses of the species, but not necessarily those showing larger amount of uses are more significant, because there are species that have a specific use and this causes them to be in a preponderant spot within the culture, as well as being considered irreplaceable; e.g. holy cross flower ('flor de la Santa Cruz', Oncidium sphacelatum), which is used solely and exclusively during May $3^{\text {rd }}$, the virgin flower ('flor de la virgen', Euphorbia pulcherrima), which is used as a decorative element in the Guadalupe Virgin ceremonies during December, or the dead flower ('cempoal/flor de muerto', Tagetes erecta), which is used in the dead's day festiveness to decorate altars and pantheons. Altogether, these aspects as a whole indicate the cultural rooting.

According to the data from the present research, to determine and try to quantify the cultural importance is sort of complex, it is considered that depending on the type of variables used, is a way to get closer to those species that are important for a particular human group. Given that there are new concepts incorporated it can be considered that the closure is being attained in order to open the guidelines to deepen into the topic, in this case referring to the biocultural key species [17-20]. It is also noticeable, that there is a wide assemble of species 
that are important culturally, authors like Rapaport [7], and Descola \& Pálsson [8] consider nature to not just being perceived as a subsistence mean, instead more like a sociability mean. To Ellen [18], traditional societies not only coexist with biodiversity, that is, nature is seen as a group of living organisms that have a symbolic use and value integrated in a complex worldview, therefore, species with a higher biocultural relevance cannot operate in an isolated way, instead there are assembles of species.

In regards, the BKSI shows which species are included in different biocultural relevance categories; in the maximum and high biocultural relevance it is considered to allow for finding the most dominant or more relevant species at a cultural level, but taking into account the aforementioned opinions, observations and collections in field, the idea about the biocultural relevance can be supported as it operates on a group of species/ethnospecies, that is also mentioned by Platten \& Henfrey [19] by describing the key biocultural species from the anthropologic point of view. It is worth noticing that the importance of species is relative and dynamic, that is, keeps evolving, growing, incorporating and excluding species through time, influenced by economic aspects, climatic changes, resource availability, public policies (health and natural resources protection), migration, commerce and globalization [68].

Starting from the analyzed species assemble (Figure 3) and taking back the key biocultural species concept [20], it is established that the location of species with a higher biocultural relevance is given from its own nature (biological features) and the inclusion in the different cultural domains, generally integrating a species or ethnospecies assemble, where it does not necessarily state that the higher values species are the most significant in a cultural level. However, the importance in determining the uses relies in adequate management and exploitation of biodiversity. It has been observed that conservation proposals that are based on this species types are more successful [23] and it encourages biocultural conservation and ancestral knowledge of the useful species.

\section{Conclusion}

Through the application of a combination of ethnographic methods, it was possible to collect trustful information by documenting the known, perceived and utilized species by the Nahua people. From there, a methodological proposal of analysis was designed to allow for a 
significant approach to the species considered to have a higher biocultural relevance to this community from knowledge (MFR-A and AMO), the role of these species in the language (RNT), as well as the diverse employment ways (VTU/PU); which results in a combination of passive and active understanding.

The idea that species that are considered as more relevant at a cultural level is sustained, as they operate in a general way; it is not about isolated species, rather of complexes or assembles; these groups of species are not necessarily related at a biological, ecological or cultural level. Hence, selection of such species depends on various factors that need to be taken into consideration. Identified biological groups are constituted in an independent way, which indicates that there is a recognition, identification and classification of the natural environment for each human group in particular. Besides, there are no biological groups that are more important to another, all of these have the same level of importance within the culture.

It is considered important to highlight the role that key biocultural species can perform, identified as species or ethnospecies complexes or assembles that further display a higher biocultural relevance, constitute elements that these can promote, facilitate and favor the biocultural diversity as a whole. The importance of the understanding of uses and employments of the key biocultural species could aim for the ethnobiological conservation locally, helping to preserve the particular identity of every culture.

\section{List of abbreviations}

BKSI: Biocultural Key Species Index

MFR-A: Mention Frequency by Rank-Abundance

AMO: Average Mention Order

RNT: Richness Names Traditional

VTU/PU: Value Total Uses / Parts Used

O: Species Origin

S-EMF: sub-evergreen medium forest

NR: Nomenclatural Relationship 
VIL: Value Linguistic Interpretation

MaxBR: Maximum Biocultural Relevance

HBR: High Biocultural Relevance

MBR: Medium Biocultural Relevance

LBR: Low Biocultural Relevance

\section{References}

1. Hunn E. The utilitarian factor in folk biological classification. Am. Anthropol. 1982. doi.org/10.1525/aa.1982.84.4.02a00070

2. Berlin B, Boster JS, O’Neill JP. The perceptual bases of ethnobiological classification: evidence from Aguarana Jívaro ornithology. J. Ethnobiol. 1981;1(1):95-108.

3. Hunn E. A Zapotec natural history. Tree, herbs and flowers, birds, beasts, and bungs in the life of San Juan Gbëë. $1^{\text {st }}$ ed. Tucson: The University of Arizona Press; 2008.

4. Rea A. Wings in the desert a folk ornithology of the Northern Pima. $1^{\text {st }}$ ed. Tucson: The University of Arizona Press; 2008.

5. Alcorn JB. Indigenous peoples and conservation. Conserv. Biol. 1993. doi.org/10.1046/j.1523-1739.1993.07020424.x

6. Berkes F, Colding J, Folke C. Rediscovery of traditional ecological knowledge as adaptative management. Ecol Appl. 2000. doi.org/10.2307/2641280

7. Rappaport R. Cerdos para los antepasados. El ritual en la ecología de un pueblo en Nueva Guinea. $1^{\text {st }}$ ed. España: Siglo XXI Editores; 1987.

8. Descola P, Pálsson G. Naturaleza y Sociedad. $1^{\text {st }}$ ed. España: Siglo XXI Editores; 2001 . 
9. Turner NJ. "The importance of a rose": Evaluating the cultural significance of plants in Thompson and Lillooet Interior Salish. Am. Anthropol. 1988. www.jstor.org/stable/677952

10. Phillips O, Gentry AH. The useful plants of Tambopata, Peru: I. Statistical hypotheses tests with a new quantitative technique. Econ Bot. 1993a. doi.org/10.1007/BF02862203

11. Phillips O, Gentry AH. The useful plants of Tambopata, Peru: II. Additional hypothesis testing in quantitative ethnobotany. Econ Bot. $1993 \mathrm{~b}$. doi.org/10.1007/BF02862204

12. Pieroni A. Evaluation of the cultural significance of wild food botanicals traditionally consumed in Northwestern Tuscany, Italy. J. Ethnobiol. 2001;21(1):89-104.

13. Garibay-Orijel R, Caballero J, Estrada-Torres A, Cifuentes J. Understanding cultural significance, the edible mushrooms case. J Ethnobiol Ethnomed. 2007. doi:10.1186/17464269-3-4.

14. Reyes-García V, Huanca T, Vadez V, Leonard W, Wilkie D. Cultural, practical, and economic value of wild plants: a quantitative study in the Bolivian Amazon. Econ Bot. 2006. doi.org/10.1663/0013-0001(2006)60[62:CPAEVO]2.0.CO;2

15. Hoffman B, Gallaher G. Importance indices in ethnobotany. Ethnobot. Res. Appl. 2007;5:201-218.

16. Tardío J, Pardo M. Cultural importance indices: a comparative analysis based on the useful wild plants of southern Cantabria (Northern Spain). Econ Bot. 2008. doi.org/10.1007/s12231-007-9004-5

17. Garibaldi A, Turner NJ. Cultural keystone species: implications for ecological conservation and restoration. Ecol. Soc. 2004. www.ecologyandsociety.org/vol9/iss3/art1/ 
18. Ellen RF. Local knowledge and management of Sago Palm (Metroxylonsagu Rottboell) diversity in South Central Seram, Maluku, Eastern Indonesia. J. Ethnobiol. 2006. doi.org./10.2993/0278-0771(2006)26[258:LKAMOS]2.0.CO;2

19. Platten S, Henfrey T. The cultural keystone concept: insights from ecological anthropology. Hum. Ecol. 2009. doi.org/10.1007/s10745-009-9237-2

20. Ibarra TJ, Barreau A, Massardo F, Rozzi R. El cóndor andino: una especie biocultural clave del paisaje sudamericano. RCO. 2012;18(1-2):1-22.

21. Paine RT. A note on trophic complexity and community stability. AM NAT. 1969. www.jstor.org/stable/2459472

22. Davic RD. Epistemology, culture, and keystone species. Ecol. Soc. 2004. www.ecologyandsociety.org/vo19/iss3/resp1/

23. Pretty J, Adams B, Berkes F, Ferreira S, Dudley N, Hunn E, et al. The intersections of biological diversity and cultural diversity: towards integration. Conserv. Soc. 2009. doi.org/10.4103/0972-4923.58642

24. Gorenflo L, Romaine J, Mittermeier S, Walker-Painemilla K. Co-occurrence of linguistic and biological diversity in biodiversity hotspots and high biodiversity wilderness areas. PNAS. 2012. doi.org/10.1073/pnas.1117511109

25. Gavin MC, McCarter J, Mead A, Berkes F, Stepp JR, Peterson D, et al. Defining biocultural approaches to conservation. Trends Ecol Evol. 2015. doi.org/10.1016/j.tree.2014.12.005

26. Gutiérrez-Santillán TV, Moreno-Fuentes A, Sánchez-González A, Sánchez-Rojas G. Knowledge and use of biocultural diversity by nahua in the Huasteca region of Hidalgo, Mexico. EC. 2019. doi:10.15451/ec2019-06-8.07-1-31 
27. Kirchhoff P. Mesoamérica: sus límites geográficos, composición étnica y caracteres culturales. Acta Americana. 1943;1:92-107.

28. Oliver G. Viaje a la Huasteca con Guy Stresser-Péan. México: FCE-CEMCA; 2008.

29. INALI. Catálogo de las lenguas indígenas nacionales: variantes lingüísticas de México con sus autodenominaciones y referencias geoestadísticas. México: Diario Oficial de la Federación. México: Gobierno de México; 2008.

30. Puig H. Vegetación de la Huasteca (México) Estudio fitogeográfico y ecológico. Francia: Institut Francais de Recherche Scientifique pour le Développement en CooperationInstituto de Ecología A.C. Centre D’Etudes Mexicaines et Centralaméricaines; 1991.

31. Challenger A, Soberón J. Los ecosistemas terrestres. In: Capítal Natural de México, vol. I: Conocimiento actual de la naturaleza. México: CONABIO; 2008. p. 87-108.

32. INEGI. Anuario estadístico del estado de Hidalgo. México: Instituto Nacional de Estadística Geografía; 2020.

33. International Society of Ethnobiology. http://ethnobiology.net/codeofethics/. 2006.

34. Albuquerque UP, Cunha L, Lucena R, Alves RRN. Methods and techniques in ethnobiology and ethnoecology. 1ed. New York: Springer; 2014.

35. Bernard HR. Research methods in anthrropology: qualitative and quantitative approaches. 4ed. Lanham-NY-Toronto-Oxford: Altamira Press; 2006.

36. Trotter RT, Needle RH, Goosby E, Bates C, Singer M. A methodological model for rapid assessment, response, and evaluation: The RARE Program in Public Health. Field Methods. 2001. doi.org/10.1177/1525822X0101300202

37. Brewer D. Supplementary interviewing techniques to maximize output in free listing tasks. Field Methods. 2002. doi.org/10.1177/1525822X02014001007 
38. Quinlan M. Considerations for collecting free lists in the field: examples from ethnobotany. Field Methods. 2005. doi.org/10.1177/1525822X05277460

39. Bautista-Nava E. Taxonomia y conocimiento tradicional de Cantharellus F. (Fungi, Cantharellaceae) en el noreste del estado de Hidalgo (Thesis Degree). México: Universidad Autónoma del Estado de Hidalgo; 2007.

40. Isidoro-Reséndiz DG. Importancia cultural de los hongos comestibles en el municipio de Tepeguacán de Guerrero, Hidalgo (Thesis Degree). México: Universidad Autónoma del Estado de Hidalgo; 2011.

41. Puig H. 1991. Vegetación de la Huasteca (México) estudio fitogeográfico y ecológico. Francia: Institut Francais de Recherche Scientifique pour le Développement en Cooperation-Instituto de Ecología A.C.-Centre D'Etudes Mexicaines et Centralaméricaines; 1991

42. Luna-Vega I, Ocegueda S, Alcántara O. Florística y notas biogeográficas del bosque mesófilo de montaña del municipio de Tlanchinol, Hidalgo, México. Anales del Instituto de Biología, Serie Botánica. 1994;65:31-62.

43. Pennington TD, Sarukhán J. Árboles tropicales de México, manual para la identificación de las principales especies. 1ed. México: Universidad Nacional Autónoma de México-Fondo de Cultura Económica; 2005.

44. Pérez-Escandón, Villavicencio-Nieto MA, Ramírez-Aguirre A. Lista de plantas útiles del estado de Hidalgo. México: Universidad Autónoma del Estado de Hidalgo; 1994.

45. Álvarez F, Villalobos JL, Armendariz G, Hernández C. Relación biogeográfica entre cangrejos dulceacuícolas y acociles a lo largo de la zona mexicana de transición: revaluación de la hipótesis de Rodríguez (1986). Rev. Mex. Biodivers. 2012. doi.org./10.7550/rmb. 28230 
46. Correa-Sandoval A. Gastrópodos terrestres del Noreste de México. Rev. Biol. Trop. 2003;51(Suppl. 3):507-522.

47. Bueno-Villegas J. Diplópodos: los desconocidos formadores del suelo. Biodiversitas. 2012;102:1-5.

48. González-Rodríguez KA, Ramírez-Pérez A, Sánchez-Barrera E, Montaño-Campos SE. Los peces de la Huasteca Hidalguense. México: Universidad Autónoma del Estado de Hidalgo-Fondo Mixto CONACyT-Gobierno del Estado de Hidalgo; 2010.

49. Miller RR. Peces dulceacuícolas de México. D.F.: CONABIO-Sociedad Ictiológica Mexicana A.C.-El Colegio de la Frontera Sur-Consejo de los Peces del Desierto MéxicoEstados Unidos; 2012.

50. Ramírez-Bautista A, Hernández-Salinas U, Mendoza-Quijano F, Cruz-Elizalde R, Stephenson BP, Vite-Silva VD Leyte-Manrique A. Lista anotada de los anfibios y reptiles. México: Universidad Autónoma del Estado de Hidalgo.CONABIO; 2010.

51. Howell SNG, Webb S. A Guide to the birds of Mexico and Northern Central America. Oxford: Oxford University Press; 1995.

52. Martínez-Morales M. Avifauna del bosque mesófilo de montaña del noreste de Hidalgo, México. Rev. Mex. Biodiv. 2007;78:149-162.

53. Martínez-Morales MA, Ortiz-Pulido R, De-la-Barreda B, Zuria IL, Bravo-Cadena, Valencia-Herverth J. Hidalgo. In: Ortiz-Pulido R, Navarro-Sigüenza A, Gómez-de-Silva H, Rojas-Soto O, Peterson TA, editors. Avifaunas Estatales de México. México: CIPAMEX; 2007. p. 49- 95.

54. Ceballos G, Oliva G (editors) Los Mamíferos silvestres de México. México: Comisión Nacional para el Conocimiento y Uso de la Biodiversidad- Fondo de Cultura Económica; 2005. 
55. Ryan GW, Nolan JM, Yoder PS. Successive free listing: Using multiple free lists to generate explanatory models. Field Methods. 2000. doi.org/10.1177/1525822X0001200201

56. Amorozo MCM, Gély AL. Uso de plantas medicinais por caboclos do Baixo Amazonas Barcarena, PA, Brasil. Boletim do Museu Paraense Emílio Goeldi, Série Botânica. 1988;4(1): 47-131.

57. Moreno CE. Métodos para medir la biodiversidad. Zaragoza: Manuales y Tesis SEA; 2001.

58. Freire KMF, Pauly D. Richness of common names of brazilian marine fishes. J. Ethnobiol. 2005. doi.org/10.2993/0278-0771(2005)25[279:ROCNOB]2.0.CO;2

59. Byg A, Balslev H. Diversity and use of palms in Zahamena, eastern Madagascar. Biodivers. Conserv. 2001. doi.org/10.1023/A:1016640713643

60. Gómez-Beloz A. Plant use knowledge of the Winikina Warao: the case for questionnaires in ethnobotany. 2002. Econ Bot. www.jstor.org/stable/4256576

61. Hammer Ø, Harper DAT, Ryan PD. PAST: Paleontological statistics software package for education and data analysis. Palaeontologica Electronica. 2001. doi:10.1017/CBO9781107415324.004

62. Cocks ML, Dold AP. Cultural significance of biodiversity: the role of medicinal plants in urban African cultural practices in the eastern cape, South Africa. J. Ethnobiol. 2006. doi.org/10.2993/0278-0771(2006)26[60:CSOBTR]2.0.CO;2

63. Caballero N, Mapes C. Gathering and subsistence patterns among the Purepecha indians of Mexico. J. Ethnobiol. 1985;5(1):31-47.

64. Lampman A. General principles classification among the Tzeltal maya of Chiapas, Mexico. J. Ethnobiol. 2007. doi.org/10.2993/0278-0771(2007)27[11:GPOECA]2.0.CO;2 
65. Guzmán G. Diversity and use of traditional Mexican fungi: A review. Int J Med Mushr. 2008. doi.org/10.1615/IntJMedMushr.v10.i3.20

66. Ruán-Soto F, Garibay-Orijel R, Cifuentes J. Process and dynamics of traditional selleing wild edible mushrooms in tropical Mexico. J Ethnobiol Ethnomed. 2006. doi.org/10.1186/1746-4269-2-3

67. Mejenes-López SMA, Hernández-Bautista M, Barragán-Torres J, Pacheco J. Los mamíferos del estado de Hidalgo, México. THERYA. 2010. doi.org/10.12933/therya-10-19

68. Lira R, Casas A, Rosas-López R, Paredes-Flores M, Pérez-Negrón E, Rangel-Landa $\mathrm{S}$, et al. Traditional knowledge and useful plant richness in the Tehuacán-Cuicatlán Valley, Mexico. Econ Bot. 2009. doi.org/10.1007/s12231-009-9075-6 


\section{Supplementary Files}

This is a list of supplementary files associated with this preprint. Click to download.

- SupplementaryMaterial.xlsx 